\title{
Rattlers' contribution to granular plasticity and mechanical stability.
}

\author{
Antoine Wautier ${ }^{\mathrm{a}, \mathrm{b}, \mathrm{c}, *}$, Stéphane Bonelli ${ }^{\mathrm{b}}$, François Nicot $^{\mathrm{c}}$ \\ ${ }^{a}$ AgroParisTech-ENGREF, 19 avenue du Maine, 75732 Paris, France. \\ ${ }^{b}$ Irstea UR RECOVER, 3275 Rte Cézanne, CS 40061, 13182 Aix-en-Provence \\ Cedex 5, France. \\ ${ }^{c}$ Université Grenoble Alpes, Irstea, UR ETGR, 2 rue de la Papeterie-BP 76, \\ F-38402 St-Martin-d'Hères, France.
}

\begin{abstract}
The mechanical behavior of granular materials is widely governed by microstructure reorganizations. This constant evolution of the microscale geometry is often taken into account directly at the macroscale within the plasticity context, and more particularly non-associated plasticity. In this paper we propose to revisit the non-associated plastic behavior of granular materials with respect to material instability assessment in terms of the loss of positiveness of the second-order work. It is shown that large incremental plastic strain is a necessary condition for the existence of mechanical instability. The ability of a material to develop substantial plastic incremental strain is then related to the existence of rattlers, i.e., free particles that get jammed into force chains when existing contact networks fail to adapt to incremental loadings. This link between rattlers, plastic deformation and the vanishing of the second-order work is explicitly derived i) from the micro-formulation of the second-order work and ii) directly from discrete element simulations.
\end{abstract}

Keywords: Stability and bifurcation, Plastic strain, Second-order work, Granular material, Rattlers

*Corresponding author: antoine.wautier@irstea.fr 


\section{Introduction}

From a microscopic point of view, a wide variety of materials fall within the family of granular materials. However, for most practical applications, their discrete nature is not accounted for directly but is hidden behind a macroscopic continuum description of their mechanical behavior. It is inconceivable, for instance, to model atoms in a metal beam or sand grains in earth dams and dikes. This change in paradigm between discrete and continuum frameworks is firmly based on the notion of scale separation, i.e., on the fact that the macroscale of interest is very large compared to the size of the grains. At the macroscale, irreversible microscale grain rearrangements are therefore usually accounted for by plastic theory as long as they remain rateindependent. For frictional granular materials such as soils or rocks, it is well established that the normality of the flow rule as proposed by Drucker and Prager (1952) does not hold, and the plastic behavior is non-associated. For such materials, the volume change predicted by an associated flow rule is indeed significantly larger than that observed in experiments with soils or rocks. To account for these observations, the choice of a potential surface different from the yield surface is often used to accommodate a much smaller volume change. Even if recent phenomenological models incorporate microstructure features through choices in the modeling functions and the state variables (see for instance Tashman et al. (2005); Lai et al. (2009); Yuanming et al. (2010); Zhu et al. (2016c)), the validation of these models is mostly based on macroscopic results. Surprisingly, the underlying microscopic hypotheses are hardly ever validated directly from grain scale simulations or measurements, making this an issue that today remains open to debate. In this respect, discrete element simulations performed on a representative elementary volume of granular material offer a possibility to investigate the effect of certain micro-mechanisms on macroscopic features of non-associated plastic models such as the flow rule direction and plastic strain intensity.

As reviewed by Darve and Laouafa (2000) for instance, the non-associated character of the flow rule imposes that the tangent constitutive tensor does not respect the so-called major symmetry. Therefore, the tangent elastoplastic matrix linking incremental stress and strain (in vector notations) is no longer symmetric (Griffiths and Willson, 1986). Since the existence of material instability is strongly related to the singularities of this matrix, the classes of bifurcations that can be expected are much more diverse than in as-

sociated plasticity. This non-symmetry explains why instability in frictional 
granular materials can be observed before reaching the plastic limit surface, provided that materials are loaded along "wedge paths" (Li and Richmond, 1997; Li and Karr, 2009). One criticism sometimes alleged to discourage the use of non-associated flow rules is that they sometimes result in indeterminate unstable solutions (Sandler and Rubin, 1987) for a number of particular loading cases (Stoughton and Yoon, 2008) or for particular elastic parameters such as large Poisson ratios (Yang et al., 2005). If we limit analyses to divergence instabilities (setting aside flutter instabilities), the second-order work criterion as introduced by Hill (1958) provides an energy interpretation for the occurrence of such instabilities. For a monotonously increasing loading, it is the first instability criterion to be encountered (Challamel et al., 2010), and other instability criteria are simply particular cases associated with specific failure mechanisms. In an updated Lagrangian formalism, the second-order work $W_{2}$ is computed from incremental variations of stress $\mathrm{d} \boldsymbol{\sigma}$ and strain $\mathrm{d} \boldsymbol{\varepsilon}$ as $W_{2}=\mathrm{d} \boldsymbol{\sigma}$ : $\mathrm{d} \boldsymbol{\varepsilon}$ where ": stands for the double contraction product. The existence of incremental loading programs leading to the vanishing of $W_{2}$ is a signature of underlying instability, because in this case at least one incremental load (including the types of boundary conditions) will result in a transition from a quasi-static to dynamic regime through a sudden release of kinetic energy (Nicot et al., 2009; Daouadji et al., 2011; Nicot et al., 2012; Wan et al., 2013, 2016; Zhang et al., 2016b; Nicot et al., 2017).

By assuming an additive decomposition of the incremental strain into an elastic part $\mathrm{d} \varepsilon^{e}$ and a plastic part $\mathrm{d} \varepsilon^{p}$, and by recalling the positive definite character of the elastic part of the second-order work $\mathrm{d} \boldsymbol{\sigma}: \mathrm{d} \boldsymbol{\varepsilon}^{e}$ (Bigoni and Hueckel, 1991), it is well known that plastic strain plays a fundamental role in the vanishing of the second-order work as $W_{2}=\mathrm{d} \boldsymbol{\sigma}: \mathrm{d} \boldsymbol{\varepsilon}^{e}+\mathrm{d} \boldsymbol{\sigma}: \mathrm{d} \boldsymbol{\varepsilon}^{p}$.

At the microscale, irreversible deformations are a consequence of particle rearrangements under the control of the local contact law and local geometry. Following the introduction of the force chain concept (Liu et al., 1995; Radjai et al., 1998), it is now well established that granular materials can be regarded as two-phase materials with only 20-30\% of the particles transmitting stresses (Peters et al., 2005; Wautier et al., 2017). The remaining loose grain fraction is not directly involved in stress transmission but plays an important role in supporting existing force chains (Zhu et al., 2016a; Tordesillas et al., 2010; Zhu et al., 2016b). More generally, the structure of granular materials may be seen as a collection of mesostructures or patterns interacting with each other and responsible for the macroscopic behavior (Dean, 2005). 
As the boundary conditions change, the population of heavily stressed grains is renewed and a phase exchange between chained and loose grains occurs. The easier this phase exchange the more versatile the granular assembly.

The aim of this paper is to provide an understanding of the relation between plastic deformation, mechanical instability and this phase exchange phenomenon with i) phenomenological macroscopic modeling, ii) micro- to macro-analytical relations and iii) three dimensional discrete element simulations. In particular we aim to investigate the role played by rattlers (i.e. particles with no contacts in the absence of gravity forces) with respect to the development of plastic strain and instability.

This paper is organized as follows. In section 2, a representative elementary volume (REV) of an idealized loose granular material is generated and subjected to a drained triaxial test. For particular stress states, the incremental constitutive behavior is modeled within non-associated elasto-plasticity. This continuum mechanics model is used to relate plastic strain development and mechanical stability from analytical considerations. From physical considerations, a conjecture on the particular role of rattlers is eventually formulated. In section 3 , the relation between mechanical stability and plastic strain obtained at the macroscale is derived directly from contact scale considerations. In the last section, the conjecture on the stabilizing role of rattlers is assessed numerically using DEM simulations.

\section{Phenomenological relation between plastic strain and mechani- cal stability in granular materials}

In this section, the link between discrete and continuum modeling of granular materials is investigated at the representative elementary volume. The mechanical behavior of a specific numerical sample is simulated using a discrete element method (DEM), and a non-associated elasto-plastic model is proposed to account for the incremental behavior. The resulting fitted phenomenological model is then used to exhibit the specific role of plasticity with respect to the existence of mechanical instability (vanishing of the secondorder work).

\subsection{Numerical modeling}

Down to the microscale, granular materials considered throughout this pa-

per are modeled as poly-disperse assemblies of spheres interacting through 


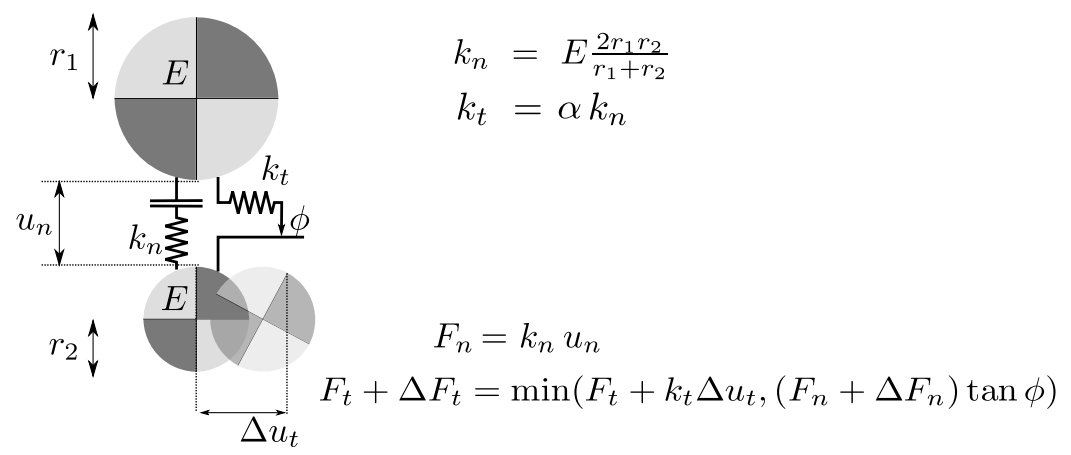

Figure 1: Elasto-frictional contact law used in DEM simulations.

the elasto-frictional contact law proposed by Cundall and Strack (1979) and illustrated in Figure 1.

Two spherical particles are said to be in contact if they overlap. A normal force $F_{n}$ is defined as proportional to the overlapping distance $\Delta u_{n}$ between the two particles where the contact normal stiffness $k_{n}$ is proportional to the material's Young modulus $E$ and to the harmonic average of the two particles radii $r_{1}$ and $r_{2}$ (see Fig. 1). In addition to the normal force, a tangential force $F_{t}$ is introduced. Contrary to its normal counterpart, this tangential force is defined in an incremental form by computing the tangential component of the incremental relative displacement $\Delta u_{t}$ at the contact point between two contacting grains (translation and rotation). $F_{t}$ is then updated according to the tangential stiffness $k_{t}=\alpha k_{n}$ expressed as a fraction $\alpha$ of its normal counterpart (see Fig. 1). The third parameter of the implemented contact law is the internal friction angle $\phi$, which defines the largest accessible ratio $F_{t} / F_{n}$ according to Mohr Coulomb theory (see Fig. 1). The input parameters used in this elasto-frictional contact law are reported in Table 1 and are similar to those used in Wautier et al. (2018).

After computing all inter-particle contact forces, the induced particle displacements are integrated based on Newton's second law of motion using the DEM open source code YADE (Šmilauer et al., 2015). In all presented simulations, gravity forces are neglected.

\subsection{Sample preparation and mechanical testing}

The sample preparation presented here is similar to Wautier et al. (2018). A cubic assembly of spheres is generated randomly with a uniform radius distribution between $r_{\min }$ and $r_{\max }=3.5 r_{\min }$. After generating a cloud 
Table 1: Mechanical parameters used in the elasto-frictional contact law implemented in YADE. The normal $\left(k_{n}\right)$ and tangential $\left(k_{t}\right)$ stiffnesses are computed as recalled in Figure 1

\begin{tabular}{ll}
\hline Parameters & Value \\
\hline Density & $3,000 \mathrm{~kg} \cdot \mathrm{m}^{-3}$ \\
Young modulus $(E)$ & $356 \mathrm{MPa}$ \\
Stiffness ratio $(\alpha)$ & 0.42 \\
Inter-particle friction angle $(\phi)$ & $35^{\circ}$ \\
Particle-wall friction angle & $0^{\circ}$ \\
Number of particles & 10,000 \\
\hline
\end{tabular}

of 10,000 non-overlapping spheres, the particles are gradually inflated while retaining the bounding walls. This radius expansion procedure is stopped when a confining pressure of $20 \mathrm{kPa}$ is reached. During this process, the inter-particle friction angle is maintained at $35^{\circ}$ in order to prepare a loose granular material with a void ratio $e=0.73$. Associated with the generated cubic grain assembly, a Cartesian coordinate system $\left(\boldsymbol{e}_{\boldsymbol{x}}, \boldsymbol{e}_{\boldsymbol{y}}, \boldsymbol{e}_{\boldsymbol{z}}\right)$ is defined such that the axis directions coincide with the edges of the cube.

From the equilibrium state obtained, a drained triaxial loading is then imposed on the sample by i) moving the bounding walls until an isotropic confining stress of $\sigma_{0}=100 \mathrm{kPa}$ is reached and then ii) imposing a vertical compression strain rate $\dot{\varepsilon}_{z z}=0.01 \mathrm{~s}^{-1}$. According to earlier numerical studies, this strain rate should be sufficiently small so that the loading can be considered as quasi-static (Hadda et al., 2013; Nicot et al., 2012).

Throughout this paper the classical soil mechanics conventions are adopted with compressions counted as positive. The homogeneous Cauchy stress tensor $\boldsymbol{\sigma}$ is defined at the representative elementary volume (REV) scale by computing the surface average of the forces applied to the bounding walls. The sample stress state is then characterized using the stress ratio $\eta$ computed as the ratio between the deviatoric stress $q$ and the mean pressure $p$ :

$$
\left\{\begin{aligned}
\eta & =\frac{q}{p} \\
q & =\sqrt{\frac{3}{2} \boldsymbol{\sigma}_{\mathrm{dev}}: \boldsymbol{\sigma}_{\mathrm{dev}}} \\
\boldsymbol{\sigma}_{\mathrm{dev}} & =\boldsymbol{\sigma}-p \mathbf{1} \\
p & =\frac{1}{3} \operatorname{Tr}(\boldsymbol{\sigma})
\end{aligned}\right.
$$

where 1 stands for the identity tensor. 

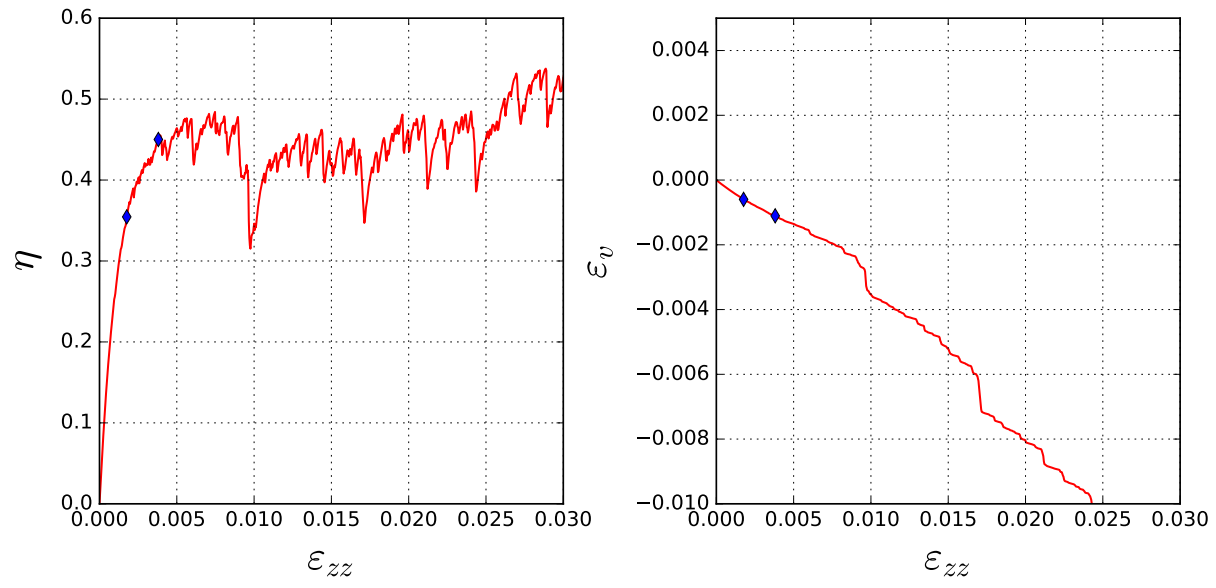

Figure 2: Drained triaxial test response similar to (Wautier et al., 2018). The diamonds correspond to the two mechanical states considered for the stability analysis in Section 4.

Based on the relative variations of the size of the whole sample, a macroscopic homogeneous strain tensor $\varepsilon$ is defined from the bounding wall displacements. To be consistent with the second-order work definition $W_{2}=\mathrm{d} \boldsymbol{\sigma}$ : $\mathrm{d} \boldsymbol{\varepsilon}$, compaction is counted positive. The volumetric strain is defined as:

$$
\varepsilon_{v}=\operatorname{Tr}(\varepsilon)
$$

In Figure 2, the stress ratio and volumetric strain responses are shown. A typical contractive behavior is observed given that both $\eta$ and $\varepsilon_{v}$ monotonously increase with the vertical compaction $\left(\varepsilon_{z z}>0\right)$. This behavior is typical of non-compacted granular material.

\subsection{Fitting a phenomenological non-associated elastoplastic model}

Throughout this subsection the sample saved at a stress ratio $\eta=0.45$ during the triaxial loading path shown in Figure 2 is considered. The incremental response of the sample is assumed to obey a classical non-associated elastoplastic relation. In this case an incremental strain $\mathrm{d} \varepsilon$ corresponding to an incremental stress $\mathrm{d} \boldsymbol{\sigma}$ can be additively broken down into an elastic part $\mathrm{d} \varepsilon^{e}$ and a plastic part $\mathrm{d} \varepsilon^{p}$ such that $\mathrm{d} \boldsymbol{\varepsilon}=\mathrm{d} \boldsymbol{\varepsilon}^{e}+\mathrm{d} \boldsymbol{\varepsilon}^{p}$. This is indeed an approximation as rigorously speaking this additive decomposition does not hold (Nicot and Darve, 2006b).

To extract the elastic part $\mathrm{d} \boldsymbol{\varepsilon}^{e}$ of the total incremental strain, one of the following two approaches found in the literature can be used: 
- by successively imposing an incremental stress loading and unloading in the same direction. The residual strain is then considered to be equal to the plastic strain (Bardet, 1994; Kishino, 2003). This method is based on the hypothesis that only reversible deformations occur during the unloading and that no elastic unloading is prevented because of grain rearrangements during the loading phase.

- by performing the same incremental stress loading twice with two different contact friction angles: the material friction angle $\phi$ and a nondissipative friction angle of $90^{\circ}$. This method prevents any plastic dissipation through friction when $\phi=90^{\circ}$ but does not prevent contact gain and loss which could result in non-reversible microstructure changes (kinetic energy released form these events is partly dissipated through numerical damping). However, these irreversible changes do not impact the macroscopic behavior significantly as long as the amplitude of the loading increment remains small. Under this condition the observed strain can be considered to be fully elastic (Nicot and Darve, 2007a; Sibille et al., 2009; Calvetti, 2003).

Following the work of Nicot et al. (2007) and Calvetti (2003), the second method was used since no hysteresis was observed while performing incremental loading and unloading with a friction angle of $90^{\circ}$.

A systematic way to track the incremental behavior of a given material consists in performing a directional analysis as introduced by Gudehus (1979). By imposing, for instance, stress probes in different directions in the stress space and recording the corresponding strain responses, the incremental behavior is then given by the implicit relation between incremental stress and incremental strain. As previously shown by Nicot and Darve (2007a), the existence of a flow rule in granular materials is limited to axisymmetric loading conditions. As a result, the directional analyses performed in this paper are restricted to the Rendulic plane $\left(\sqrt{2} \mathrm{~d} \sigma_{x x}, \mathrm{~d} \sigma_{z z}\right)$ (this corresponds to the plane $\mathrm{d} \sigma_{x x}=\mathrm{d} \sigma_{y y}$ in the stress space). By considering $\sqrt{2} \mathrm{~d} \sigma_{x x}$ instead of $\mathrm{d} \sigma_{x x}$, scalar products in the Rendulic plane correspond to scalar products computed directly in the three dimensional stress space $\left(\mathrm{d} \sigma_{x x}, \mathrm{~d} \sigma_{y y}, \mathrm{~d} \sigma_{z z}\right)$. In the inset of Figure 3, incremental elastic strains are plotted in the axisymmetric plane $\left(\sqrt{2} \mathrm{~d} \varepsilon_{x x}^{e}, \mathrm{~d} \varepsilon_{z z}^{e}\right)$ while incremental stress probes describe a circle in the Rendulic plane $\left(\sqrt{2} \mathrm{~d} \sigma_{x x}, \mathrm{~d} \sigma_{z z}\right)$. 


$$
\left\{\begin{array}{cl}
\sqrt{2} \mathrm{~d} \sigma_{x x}=\sqrt{2} \mathrm{~d} \sigma_{y y} & =\|\mathrm{d} \boldsymbol{\sigma}\| \cos \theta \\
\mathrm{d} \sigma_{z z} & =\|\mathrm{d} \boldsymbol{\sigma}\| \sin \theta \\
\|\mathrm{d} \boldsymbol{\sigma}\| & =\sqrt{\mathrm{d} \sigma_{z z}^{2}+2 \mathrm{~d} \sigma_{x x}^{2}}
\end{array}\right.
$$

where $\|\mathrm{d} \boldsymbol{\sigma}\|=5 \mathrm{kPa}$ and $\theta \in[0,2 \pi]$.

Extending Bardet's 2D work (Bardet, 1994), the incremental elastic strain envelope shown in Figure 3 is typical of an incremental anisotropic elastic behavior. Let us assume that the sample behaves as a transverse isotropic material. This hypothesis is based on the following two points: i) the sample preparation procedure results in an isotropic sample and ii) the triaxial loading introduces an anisotropy in the vertical direction (see autocorrelation distances in Wautier et al. (2017), for instance). Thus, in the Rendulic plane, the constitutive matrix relating the incremental strain to the imposed incremental stress reads as:

$$
\left(\begin{array}{c}
\sqrt{2} \mathrm{~d} \varepsilon_{x x}^{e} \\
\mathrm{~d} \varepsilon_{z z}^{e}
\end{array}\right)=\left(\begin{array}{cc}
\frac{1-\nu_{h}}{E_{h}} & -\sqrt{2} \frac{\nu_{h v}}{E_{h}} \\
-\sqrt{2} \frac{\nu_{h v}}{E_{h}} & \frac{1}{E_{v}}
\end{array}\right) \cdot\left(\begin{array}{c}
\sqrt{2} \mathrm{~d} \sigma_{x x} \\
\mathrm{~d} \sigma_{z z}
\end{array}\right)
$$

where $E_{h}$ and $E_{v}$ are the Young modulus in the horizontal and vertical directions respectively and $\nu_{h}$ and $\nu_{h v}$ are the Poisson ratios between the horizontal directions and between the horizontal and vertical directions, respectively. While d $\boldsymbol{\sigma}$ describes a circle in the Rendulic plane (Equation (3)), d $\boldsymbol{\varepsilon}$ describes a tilted ellipse in the axisymmetric plane $\left(\sqrt{2} \mathrm{~d} \varepsilon_{x x}^{e}, \mathrm{~d} \varepsilon_{z z}^{e}\right)$ with the following parametric equation:

$$
\left\{\begin{array}{rl}
\sqrt{2} \mathrm{~d} \varepsilon_{x x}^{e}=\sqrt{2} \mathrm{~d} \varepsilon_{y y}^{e} & =\|\mathrm{d} \boldsymbol{\sigma}\|\left[\frac{1-\nu_{h}}{E_{h}} \cos \theta-\frac{\sqrt{2} \nu_{h v}}{E_{h}} \sin \theta\right] \\
\mathrm{d} \varepsilon_{z z}^{e} & =\|\mathrm{d} \boldsymbol{\sigma}\|\left[-\frac{\sqrt{2} \nu_{h v}}{E_{h}} \cos \theta+\frac{1}{E_{v}} \sin \theta\right]
\end{array} .\right.
$$

By minimizing the quadratic error between the numerical points and Equation (5), a very good prediction of the numerical results is achieved by choosing $E_{h}=25.2 \mathrm{MPa}, E_{v}=32.5 \mathrm{MPa}$ and $\nu_{h}=\nu_{h v}=\nu=0.31$. The resulting fitted model is shown in Figure 3.

As summarized in Figure 6, the elastic incremental behavior is accounted for in the Rendulic plane through three material constants $E_{h}, E_{v}$ and $\nu$. It must be underlined here that the choice $\nu_{h}=\nu_{h v}$ is arbitrary and has no reason to hold outside of the Rendulic plane. Indeed, the three independent material constants at stake in Equation (4) are $\frac{1-\nu_{h}}{E_{h}}, \frac{\nu_{h v}}{E_{h}}$ and $E_{v}$. However, 


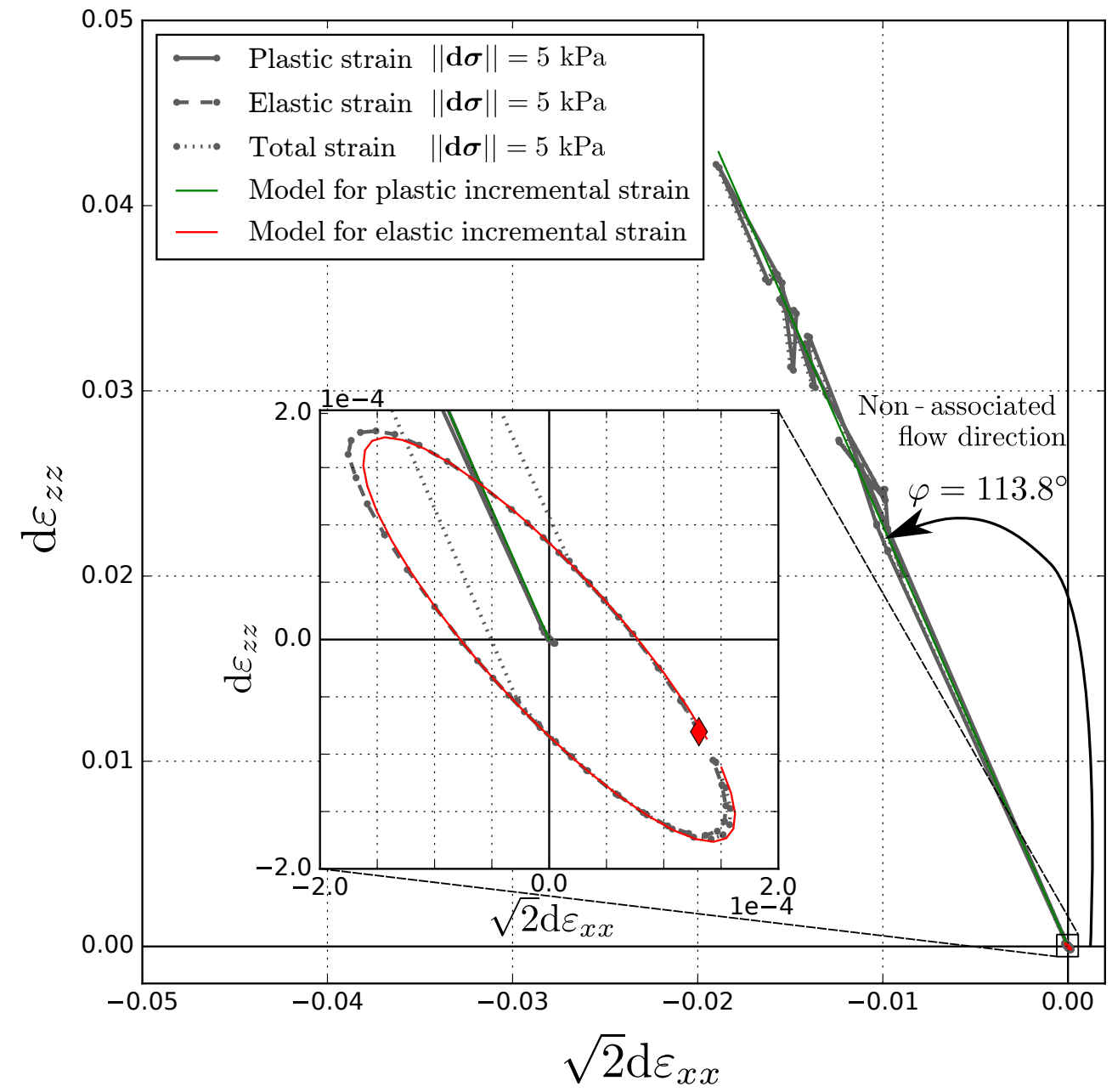

Figure 3: Incremental strain responses in the axisymmetric plane for the sample corresponding to $\eta=0.45$ while incremental stress probes describe a circle in the Rendulic plane (Equation (3)). The total incremental strain (dotted line) is broken down into its elastic (dashed line) and plastic parts (solid line). The point corresponding to $\theta=0$ is shown with a diamond in the inset plot. Fitted elastic and plastic models for incremental strains are shown with thin solid lines. 


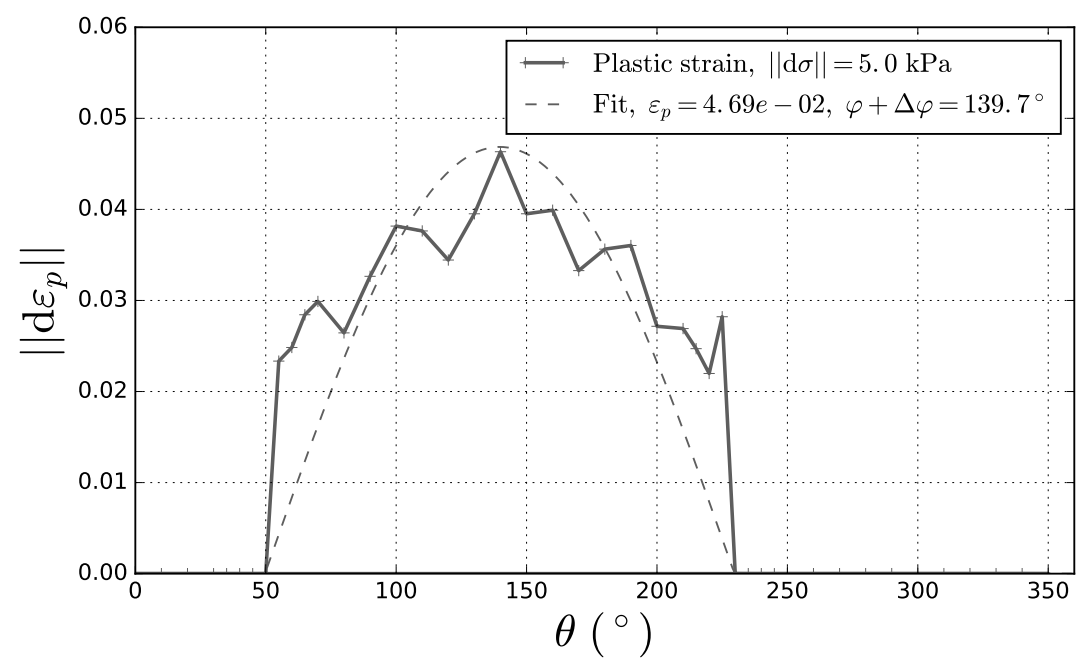

Figure 4: Evolution of the norm of the incremental plastic strain $\left\|\mathrm{d} \varepsilon^{p}\right\|$ with respect to the loading angle $\theta$. Numerical results are shown as a solid line while the fitted truncated cosine function is shown as a dashed line.

assuming $\nu_{h}=\nu_{h v}$ is though convenient to benchmark the present material parameters to the classical Young modulus obtained for uniformly distributed loose sands that range from 10 to $30 \mathrm{MPa}$. Based on the load history, the vertical compaction induced the building of force chains mainly aligned in this direction (Wautier et al., 2017). As a result, the sample is expected to be stiffer in the vertical direction than in the horizontal one which is consistent with $E_{v}>E_{h}$.

As discussed in Bardet (1994) in 2D, the inclination of the ellipse is linked to the ratio between $E_{h}$ and $E_{v}$. For an isotropic material, the inclination angle should be of $-54.7^{\circ}$ (instead of $45^{\circ}$ in $2 \mathrm{D}$ ) whereas in our case an inclination of $-50.4^{\circ}$ is measured (the derivations of these angles are not given here but are simply consequences of Equation (5)).

By subtracting the incremental elastic strain from the total strain, the plastic contribution is then recovered. In Figure $3, \mathrm{~d} \varepsilon^{p}$ is plotted in the axisymmetric plane, whereas the norm $\left\|\mathrm{d} \varepsilon^{p}\right\|$ is plotted against the stress probe angle $\theta$ in Figure 4.

In Figure 3, the plastic strain concentrates in a single direction $\varphi$ in the strain axisymmetric plane whatever the loading direction $\theta$. This indicates a typical flow rule the direction of which is $\varphi$ in the plane $\left(\sqrt{2} \mathrm{~d} \varepsilon_{x x}, \mathrm{~d} \varepsilon_{x x}\right)$. In Figure 4, the plastic flow intensity may be approximated by a truncated 


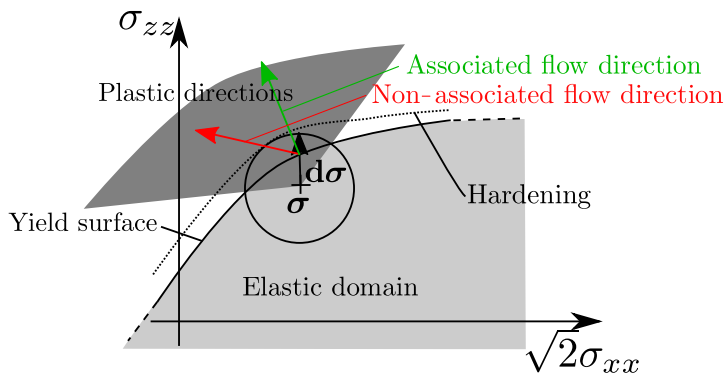

(a) Non-associated flow rule

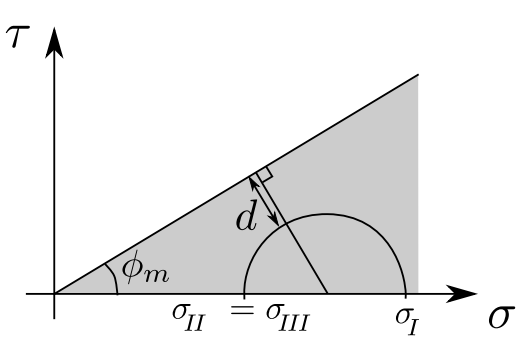

(b) Distance to Mohr-Coulomb limit

Figure 5: (a) Illustration of an associated and non-associated flow rule in the Rendulic plane. Starting from a state $\boldsymbol{\sigma}$, an incremental load $\mathrm{d} \boldsymbol{\sigma}$ is applied. If the plastic limit is crossed, a plastic increment exists and the plastic limit may move if hardening occurs. (b) Illustration of the distance $d$ to the Morh-Coulomb plastic limit using Mohr circle.

cosine function with a maximum intensity $\varepsilon_{p}$ in the stress loading direction $\theta=\varphi+\Delta \varphi$ normal to the plastic yield surface:

$$
\left\{\begin{array}{cl}
\sqrt{2} \mathrm{~d} \varepsilon_{x x}^{p}=\sqrt{2} \mathrm{~d} \varepsilon_{y y}^{p} & =\varepsilon_{p} \max (0, \cos (\theta-\varphi-\Delta \varphi)) \cos (\varphi) \\
\mathrm{d} \varepsilon_{z} z^{p} & =\varepsilon_{p} \max (0, \cos (\theta-\varphi-\Delta \varphi)) \sin (\varphi)
\end{array} .\right.
$$

By minimizing the quadratic error between the numerical points and Equation (6), a reasonable prediction of the numerical results is achieved by choosing $\varphi=113.8^{\circ}, \Delta \varphi=25.9^{\circ}$ and $\varepsilon_{p}=4.6910^{-2}$. The resulting fitted model is included in Figure 3.

The non-zero value for $\Delta \varphi$ is a signature of the non-associated character of the flow rule (Nicot and Darve, 2007b; Sibille et al., 2009). Indeed, in the case of associated plasticity, the direction of the plastic flow rule is given by the perpendicular direction to the yield surface in the stress space, as illustrated in Figure 5 (a). If the reference stress state is close enough to the plastic limit, the angular range of incremental loadings $\mathrm{d} \boldsymbol{\sigma}$ able to activate plasticity is close to $180^{\circ}$ (see Figure 5 (a)). In Figure 4, the loading angles for which $\left\|\mathrm{d} \varepsilon^{p}\right\| \neq 0$ are such that $\theta \in\left[49.7^{\circ}, 229.7^{\circ}\right]$. The reference state $\eta=0.45$ therefore belongs to the yield surface. As a result, in our case $\varphi+\Delta \varphi$ corresponds to the normal to the plastic limit surface, which is equal to $139.7^{\circ}$. The observed flow direction in Figure 3 is equal to $\varphi=113.8^{\circ}$ and not $139.7^{\circ}$, however, which is incompatible with the existence of an associated flow rule.

If a Mohr-Coulomb plastic limit is assumed, the half-plastic plane $(\theta \in[\varphi+$ $\left.\Delta \varphi-\frac{\pi}{2}, \varphi+\Delta \varphi+\frac{\pi}{2}\right]$ can be linked to the material friction angle $\phi_{m}$ (at the 
continuum scale). Indeed, for stress states in the Rendulic plane, the distance $d$ between the Mohr-Coulomb straight line and the Mohr circle representing the stress state (see Figure $5(\mathrm{~b})$ ) reads

$$
d=\frac{\sigma_{I I I}+\sigma_{I}}{2} \sin \phi_{m}-\frac{\sigma_{I}-\sigma_{I I I}}{2} .
$$

where $\sigma_{I}=\sigma_{z z}$ and $\sigma_{I I}=\sigma_{I I I}=\sigma_{x x}$ and $\sigma_{I I I}$ are the principal stresses in decreasing order.

Remembering the fact that initially the stress state is given by a horizontal confinement $\sigma_{0}$ and a vertical stress characterized by the stress ratio $\eta$, the total stress components to be considered during the directional analysis given in Equation (3) are

$$
\left\{\begin{aligned}
\sigma_{x x} & =\sigma_{0}+\frac{\|\mathrm{d} \boldsymbol{\sigma}\|}{\sqrt{2}} \cos \theta \\
\sigma_{z z} & =\frac{2 \eta+1}{1-\eta} \sigma_{0}+\|\mathrm{d} \boldsymbol{\sigma}\| \sin \theta
\end{aligned}\right.
$$

which leads to

$$
d(\theta)=\sigma_{0} \frac{(2+\eta) \sin \phi_{m}-3 \eta}{2(1-\eta)}+\|\mathrm{d} \boldsymbol{\sigma}\| \frac{\sqrt{3-2 \sin \phi_{m}+3 \sin ^{2} \phi_{m}}}{2} \cos \left(\theta+\theta_{0}\right)
$$

with $\theta_{0}$ defined such that

$$
\left\{\begin{array}{l}
\cos \theta_{0}=\frac{1+\sin \phi_{m}}{\sqrt{3-2 \sin \phi_{m}+3 \sin ^{2} \phi_{m}}} \\
\cos \theta_{0}=\frac{\sqrt{2}\left(1-\sin \phi_{m}\right)}{\sqrt{3-2 \sin \phi_{m}+3 \sin ^{2} \phi_{m}}} .
\end{array}\right.
$$

As a result, directions corresponding to non-zero plastic increments are given by $d(\theta)<0$. When the initial state belongs to the yield surface $(d=0$ for $\|\mathrm{d} \boldsymbol{\sigma}\|=0)$ the suitable range for $\theta$ is $\left[\frac{\pi}{2}-\theta_{0}, \frac{3 \pi}{2}-\theta_{0}\right]$, whose amplitude is $180^{\circ}$. In our case, this assumption is likely to hold true as the amplitude of non-zero values for the plastic incremental strain in Figure 4 is $180^{\circ}$ which is a consequence of the hardening observed during the drained triaxial loading in Figure 2. As a result, the vanishing of $d$ for $\|\mathrm{d} \boldsymbol{\sigma}\|=0$ corresponds to a material friction angle $\phi_{m}=33.4^{\circ}$. For such a material friction angle, the half-plastic plane predicted by Equation $(9)$ is $\left[\frac{\pi}{2}-\theta_{0}, \frac{3 \pi}{2}-\theta_{0}\right]=\left[68^{\circ}, 248^{\circ}\right]$, which is slightly different from the one observed in Figure $4\left[\varphi+\Delta \varphi-\frac{\pi}{2}, \varphi+\right.$ $\left.\Delta \varphi+\frac{\pi}{2}\right]=\left[50^{\circ}, 230^{\circ}\right]$. The difference observed might stem from the fact that the material does not follow a perfect Mohr-Coulomb plastic criterion as assumed to derive Equation (9). 


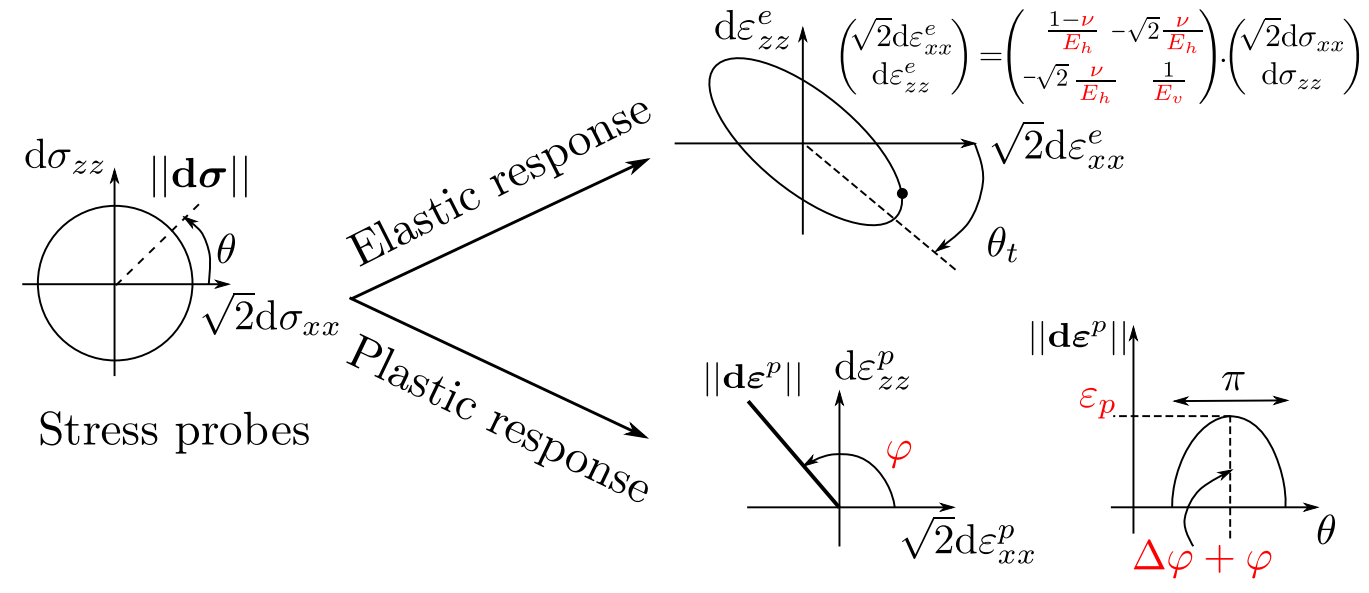

Figure 6: Non-associated elastoplastic fitted response in the incremental strain space corresponding to stress probes describing a circle in the Rendulic plane. The strain response is broken down into an elastic part characterized by three parameters and a plastic part described with three parameters.

Table 2: Fitted parameters for the incremental non-associated elastoplastic behavior of the sample saved in the mechanical state $\eta=0.45$.

\begin{tabular}{l|l} 
Elastic behavior & Plastic behavior \\
\hline$E_{h}=25.2 \mathrm{MPa}$ & $\varphi=113.8^{\circ}$ \\
$E_{v}=32.5 \mathrm{MPa}$ & $\Delta \varphi=25.9^{\circ}$ \\
$\nu=0.31$ & $\varepsilon_{p}=4.6910^{-2}$
\end{tabular}

In the end, the fitting procedure described in this subsection and summarized in Figure 6 is based on the identification of six parameters. For the sample prepared in the mechanical state $\eta=0.45$ the fitted parameters are given in Table 2.

\subsection{Plastic strain intensity influence on the vanishing of the second-order work}

The phenomenological model introduced in the previous subsection and fitted in the Rendulic plane is convenient to interpret the vanishing of the secondorder work in terms of macro variables such as the maximum level of plastic strain $\varepsilon_{p}$. Indeed, based on Equations (3), (5) and (6), in the plastic domain $\theta \in\left[\varphi+\Delta \varphi-\frac{\pi}{2} ; \varphi+\Delta \varphi+\frac{\pi}{2}\right]$, the second-order work reads 


$$
\begin{aligned}
W_{2} & =\mathrm{d} \boldsymbol{\sigma}:\left(\mathrm{d} \varepsilon^{e}+\mathrm{d} \varepsilon^{p}\right) \\
& =\|\mathrm{d} \boldsymbol{\sigma}\| \sqrt{\beta^{2}+\gamma^{2}}\left(\frac{\alpha}{\sqrt{\beta^{2}+\gamma^{2}}}+\cos \left(2 \theta-\varphi_{0}\right)\right)
\end{aligned}
$$

with

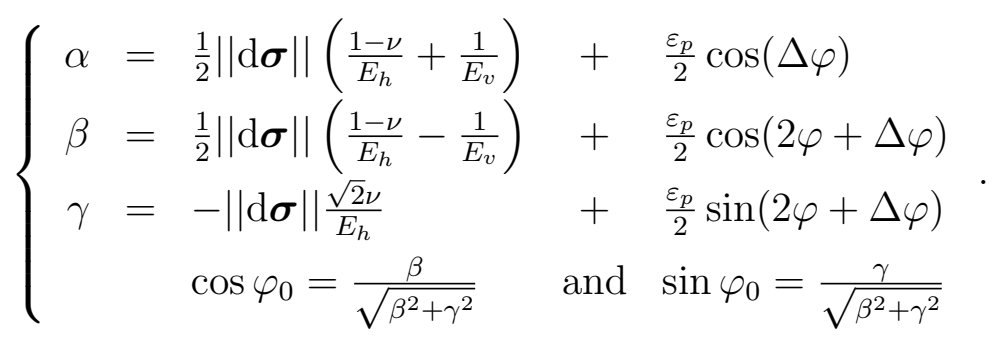

In Figure 7 the analytical expression (11) (parameters from Table 2) is compared with the DEM results for the sample corresponding to $\eta=0.45$. A circular representation is used. This representation consists in a polar plot of $1+\bar{W}_{2}(\theta)$, with $\bar{W}_{2}=\frac{\mathrm{d} \varepsilon: \mathrm{d} \boldsymbol{\sigma}}{\|\mathrm{d} \boldsymbol{\varepsilon}\|\|\mathrm{d} \boldsymbol{\sigma}\|}$ the normalized form of the second-order work. The vanishing of the second-order is then observed graphically as the polar curve $1+\bar{W}_{2}(\theta)$ intersects the disk of unit radius.

In Figure 7, the fitted model is shown to account nearly perfectly for the shape of the circular envelopes obtained numerically. The relative contribution of the elastic and plastic strains to the normalized second-order work are shown with dashed lines $\left(W_{2}=W_{2}^{e}+W_{2}^{p}\right)$. For the set of parameters in Table 2, two half-planes can be defined in which $W_{2} \simeq W_{2}^{e}$ or $W_{2} \simeq W_{2}^{p}$. The second-order work is observed to vanish in the half-plastic plane $\left[\varphi+\Delta \varphi-\frac{\pi}{2} ; \varphi+\Delta \varphi+\frac{\pi}{2}\right]$ which is a signature of material instability by divergence. The non-associated character of the flow rule is visible because the normal direction $\varphi+\Delta \varphi$ to the half-plastic plane (dashed line) differs from the direction of plastic increment in the axisymmetric plane $\varphi$ (solid line).

As a result of Equation (11), a necessary condition for having a loading direction leading to the vanishing of the second-order work reads

$$
\text { If } \exists \theta \text { such that } W_{2}(\theta)<0 \text {, then } \alpha^{2} \leq \beta^{2}+\gamma^{2} \text {. }
$$

If this condition is satisfied and if the directions $\theta$ giving $W_{2}<0$ lie in the plastic half-plane $\left[\varphi+\Delta \varphi-\frac{\pi}{2} ; \varphi+\Delta \varphi+\frac{\pi}{2}\right]$ (otherwise Equation (11) is not valid), the granular material considered is at a bifurcation point. In other words, even if the material considered is at equilibrium in the current state (defined by the geometry of its microstructure and its stress state), 


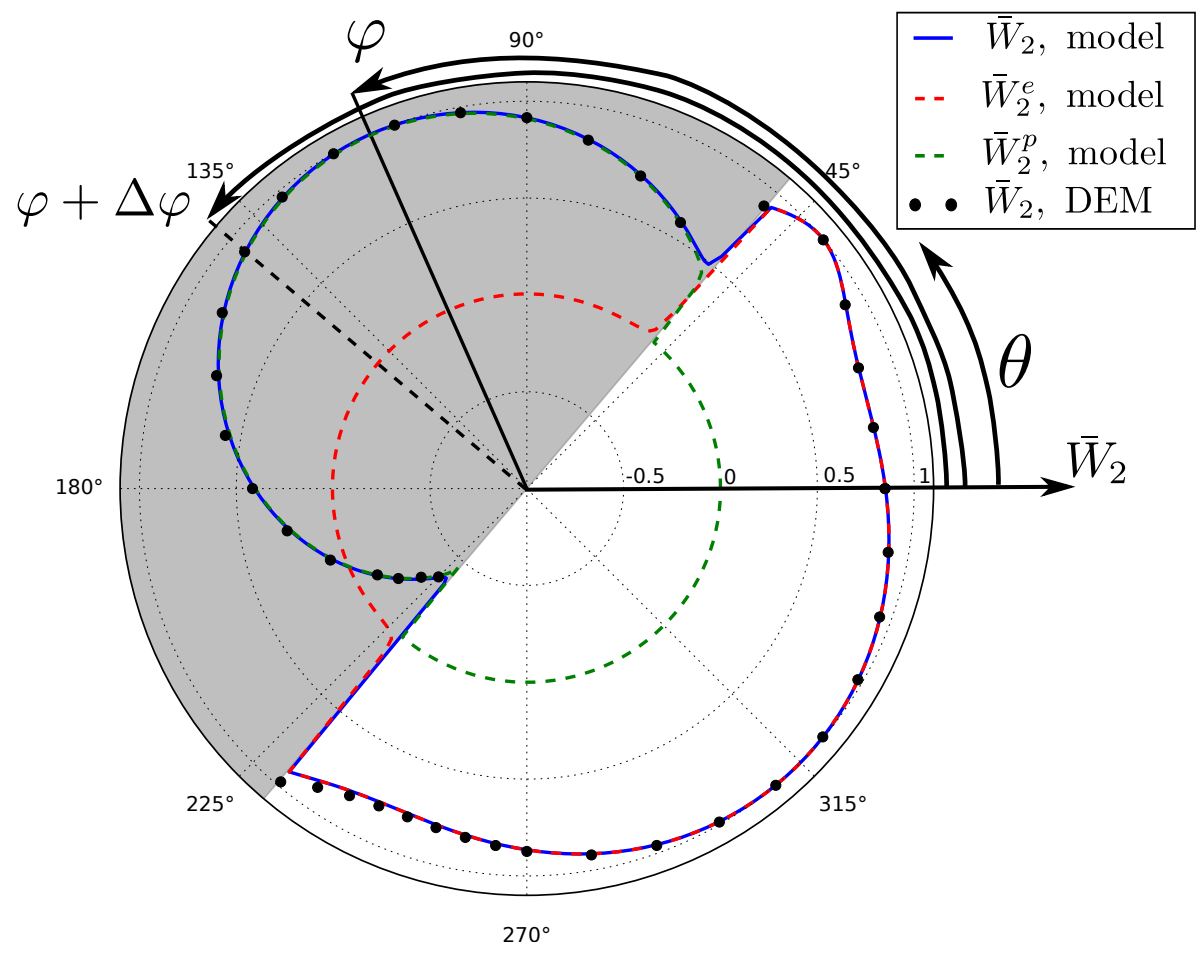

Figure 7: Comparison between analytical (solid lines) and numerical (dots) normalized second-order work envelopes for the initial sample corresponding to $\eta=0.45$. The model parameters are taken from Table 2. The relative contribution of the elastic and plastic incremental strain in the computation of $W_{2}$ are shown in dashed lines. The plastic halfplane $\left[\varphi+\Delta \varphi-\frac{\pi}{2} ; \varphi+\Delta \varphi+\frac{\pi}{2}\right]$ corresponds to the grey domain. The maximum plastic intensity direction $\varphi+\Delta \varphi$ (black dashed line) and the plastic flow direction $\varphi$ (black solid line) are also shown. 


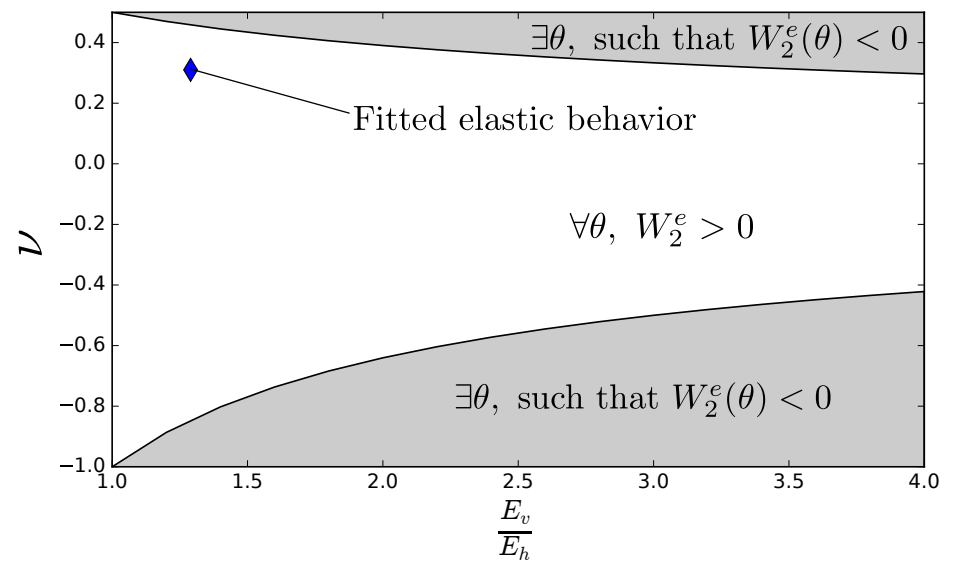

Figure 8: Phase diagram of couples $\left(\frac{E_{v}}{E_{h}}, \nu\right)$ leading to the vanishing of the elastic part $W_{2}^{e}$ of the second-order work. The point corresponding to the fitted parameters in Table 2 is indicated.

incremental loading programs exist that will lead to material failure through an abrupt increase in kinetic energy.

If we assume that the material behavior is fully elastic $\left(\varepsilon_{p}=0\right)$, then condition (13) can be written in terms of material constants $\nu$ and $E_{v} / E_{h}$ as

$$
\nu \leq-\frac{1+\sqrt{1+8 \frac{E_{v}}{E_{h}}}}{4 \frac{E_{v}}{E_{h}}} \quad \text { or } \quad \nu \geq \frac{-1+\sqrt{1+8 \frac{E_{v}}{E_{h}}}}{4 \frac{E_{v}}{E_{h}}}
$$

and is illustrated in Figure 8.

Even for a purely elastic material behavior, the vanishing of the secondorder work is possible for exotic materials for which the anisotropy $E_{v} / E_{h}$ is very large (Nicot et al., 2016) or equivalently for sufficiently large Poisson ratios (Yang et al., 2005). In our case and for usual materials for which $\nu \in[0.2,0.4]$ and $E_{v} / E_{h}<2$, no vanishing of the second-order work is expected if $\varepsilon_{p}=0$. As a result, the size of the plastic increment is expected to play an important role with respect to the vanishing of the second-order work. This sensitivity to $\varepsilon_{p}$ is explored in Figure 9 in which normalized circular envelopes of the second-order work are shown for different $\varepsilon_{p}$ values while keeping other parameters of Table 2 constant.

As expected, the value of $\varepsilon_{p}$ has a great influence on the existence and the width of the cone of instability resulting from Equations (11) and (12). The 


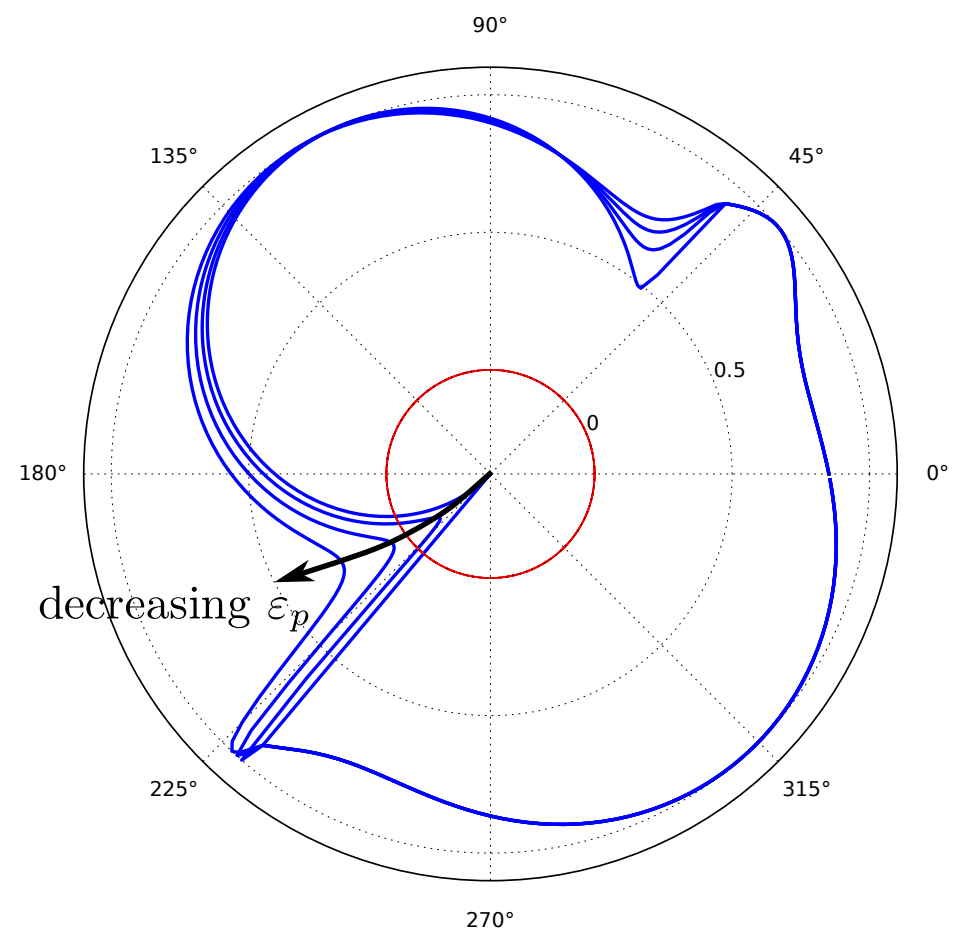

Figure 9: Analytical normalized second-order work envelopes for different plastic intensities $\varepsilon_{p} \in\left\{\varepsilon_{p}^{\circ}, \varepsilon_{p}^{\circ} / 20, \varepsilon_{p}^{\circ} / 50, \varepsilon_{p}^{\circ} / 100\right\}$ where $\varepsilon_{p}^{\circ}=4.6910^{-2}$. The six other parameters of the model are selected from Table 2 . 

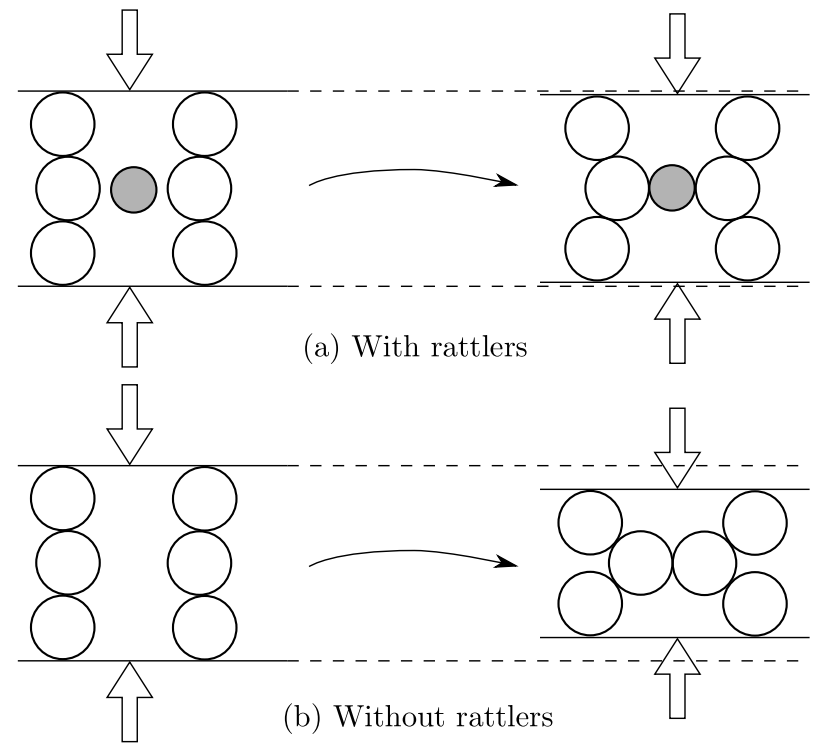

Figure 10: Schematic diagram of the particular role played by rattlers with respect to plastic strain development. Comparison between a microstructure with rattlers (a) and the same microstructure without rattlers (b).

larger $\varepsilon_{p}$, the larger the instability cone. This close relationship between the vanishing of the second-order work and the size of the plastic increments was also recently accounted for by Zhang et al. (2016a) while considering the occurrence of "strain bursts".

\subsection{A conjecture on the particular role played by rattlers}

From the results illustrated in Figure 9 combined with the schematic diagram in Figure 10, we conjecture that rattlers (particles with no contacts in absence of gravity) play the particular role of limiting the development of plastic strain when the existing contact network fails to adapt to the newly imposed mechanical loading and induces microstructure rearrangements. Given that large incremental plastic strain development is a necessary condition for the onset of macroscopic instability, rattlers play a fundamental role in mitigating material instability by divergence.

This issue will be discussed from micromechanical considerations in the following two sections. 


\section{A contact scale explanation for the stabilizing role played by rattlers}

Complementary to the previous section, the particular role played by rattlers in mitigating plastic strain development can be examined analytically and qualitatively using the micro-formulation of the second-order work introduced for granular materials (Nicot et al., 2007; Hadda et al., 2013). In this section the contact scale necessary condition to observe the vanishing of the second-order work (Nicot and Darve, 2006a; Nicot et al., 2013) is revisited in terms of rattlers influence to provide an analytical proof of the conjecture formulated in Section 2.5. This section is kept qualitative on purpose but a quantitative assessment of the role played by rattlers directly at the contact scale is proposed in Appendix A through DEM simulations.

Let $\ell^{c}$ be the branch vector connecting the centers of two contacting spherical particles at contact $c, \boldsymbol{F}^{c}$ the inter-particle contact force, $\boldsymbol{F}^{p}$ the resultant contact force on particle $p$ and $\boldsymbol{x}^{p}$ the position of its mass center. Then the volume integral of the second-order work on a sample of domain $\Omega$ can be computed from micro-quantities as

$$
\int_{\Omega} W_{2} \mathrm{~d} V=\sum_{c} \Delta \boldsymbol{F}^{c} \cdot \Delta \boldsymbol{\ell}^{c}+\sum_{p} \Delta \boldsymbol{F}^{p} \cdot \Delta \boldsymbol{x}^{p}
$$

where $\Delta$ correspond to incremental variations and $\cdot$ to the scalar product. Provided that only inertial terms can be ignored $\left(\forall p, \Delta \boldsymbol{F}^{p}=\mathbf{0}\right)$, the second term vanishes and the second-order work computed at the REV scale reads:

$$
W_{2}=\frac{1}{|\Omega|} \sum_{c} \Delta \boldsymbol{F}^{c} \cdot \Delta \boldsymbol{\ell}^{c} .
$$

Consequently a microscopic second-order work can be defined at the contact scale as $w_{2}^{c}=\Delta \boldsymbol{F}^{c} \cdot \Delta \boldsymbol{\ell}^{c}$ (Nicot and Darve, 2006a).

By further breaking down $\Delta \boldsymbol{F}^{c}$ and $\Delta \boldsymbol{\ell}^{c}$ into normal and tangential components, $w_{2}^{c}$ can be expressed as

$$
w_{2}^{c}=\Delta \boldsymbol{F}_{n}^{c} \cdot \Delta \boldsymbol{\ell}_{n}^{c}+\Delta \boldsymbol{F}_{t}^{c} \cdot \Delta \boldsymbol{\ell}_{t}^{c} .
$$

The definition of the different vectors at stake in this equation appears more clearly in Figure 11.

Using the contact law recalled in Figure 1, the first term reads $k_{n}\left\|\Delta \boldsymbol{\ell}_{n}^{c}\right\|^{2}$ and is always positive, as is the second one as long as $\left\|\boldsymbol{F}_{t}^{c}\right\| /\left\|\boldsymbol{F}_{n}^{c}\right\|<\tan \phi$. As a 


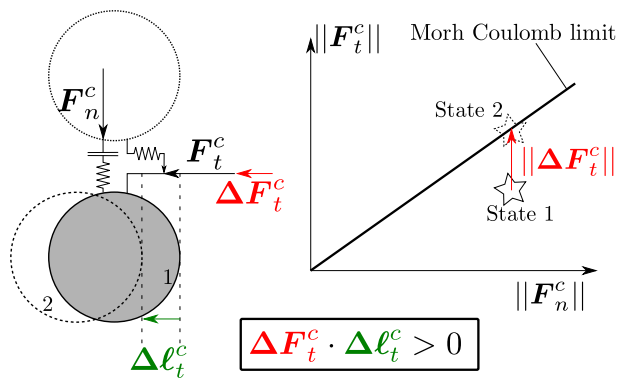

(a) Sliding through increase in $\left\|\boldsymbol{F}_{t}^{c}\right\|$

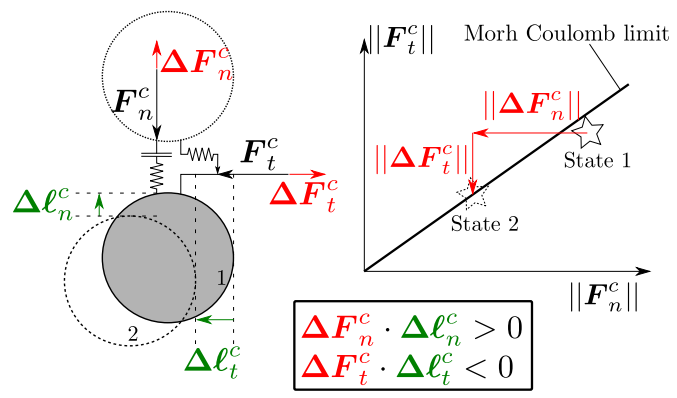

(b) Sliding through decrease in $\left\|\boldsymbol{F}_{n}^{c}\right\|$

Figure 11: Two incremental loadings leading to contact sliding with different consequences on the sign of $w_{2}^{c}$. While configuration (a) results in $w_{2}^{c}>0$, configuration (b) may result in $w_{2}^{c}<0$ depending on $\left\|\Delta \ell_{t}^{c}\right\|$.

result, $w_{2}^{c} \geq 0$ if the contact behaves elastically. If sliding occurs, the sign of $w_{2}^{c}$ is difficult to predict and two cases should be considered (see Figure 11 and Nicot and Darve (2006a); Nicot et al. (2013)).

(a) $\boldsymbol{F}_{n}^{c}$ remains constant and $\Delta \boldsymbol{F}_{t}^{c}$ is such that $\boldsymbol{F}_{n}^{c}+\boldsymbol{F}_{t}^{c}+\Delta \boldsymbol{F}_{t}^{c}$ reaches the Mohr-Coulomb limit cone. In this case the tangential relative displacement $\Delta \boldsymbol{\ell}_{t}^{c}$ is positively collinear to $\boldsymbol{F}_{t}^{c}+\Delta \boldsymbol{F}_{t}^{c}$ and for a sufficiently small increment $\Delta \boldsymbol{F}_{t}^{c}, \Delta \boldsymbol{F}_{t}^{c} \cdot \Delta \boldsymbol{\ell}_{t}^{c} \geq 0$. As a result $w_{2}^{c} \geq 0$.

(b) $\left\|\boldsymbol{F}_{n}^{c}\right\|$ decreases such that $\boldsymbol{F}_{n}^{c}+\Delta \boldsymbol{F}_{n}^{c}+\boldsymbol{F}_{t}^{c}$ violates the Mohr-Coulomb limit cone as $\left\|\boldsymbol{F}_{t}^{c}\right\|>\left(\left\|\boldsymbol{F}_{n}^{c}\right\|-\left\|\Delta \boldsymbol{F}_{n}^{c}\right\|\right) \tan \phi$. In this case, the tangential force decreases as:

$$
\Delta \boldsymbol{F}_{t}^{c}=-\left[\left\|\boldsymbol{F}_{t}^{c}\right\|-\left(\left\|\boldsymbol{F}_{n}^{c}\right\|-\left\|\Delta \boldsymbol{F}_{n}^{c}\right\|\right) \tan \phi\right] \frac{\boldsymbol{F}_{t}^{c}}{\left\|\boldsymbol{F}_{t}^{c}\right\|}
$$

whereas the relative displacement $\Delta \boldsymbol{\ell}_{t}^{c}$ is positively collinear to $\boldsymbol{F}_{t}^{c}$. In this case, the tangential term in $w_{2}^{c}$ is negative and:

$$
w_{2}^{c}=\frac{1}{k_{n}}\left\|\Delta \boldsymbol{F}_{n}^{c}\right\|^{2}-\left[\frac{\left\|\boldsymbol{F}_{t}^{c}\right\|}{\left\|\boldsymbol{F}_{n}^{c}\right\|}-\left(1-\frac{\left\|\Delta \boldsymbol{F}_{n}^{c}\right\|}{\left\|\boldsymbol{F}_{n}^{c}\right\|}\right) \tan \phi\right]\left\|\boldsymbol{F}_{n}^{c}\right\|\left\|\Delta \boldsymbol{\ell}_{t}^{c}\right\|
$$

As a result, it should be underlined that the occurrence of contact sliding is not a sufficient condition to observe the vanishing of $w_{2}^{c}$. If a contact slides under an "increase" in the tangential force, for instance, the scalar product $\Delta \boldsymbol{u}_{t} \cdot \Delta \boldsymbol{F}_{t}$ is positive. Contact sliding through normal unloading is 


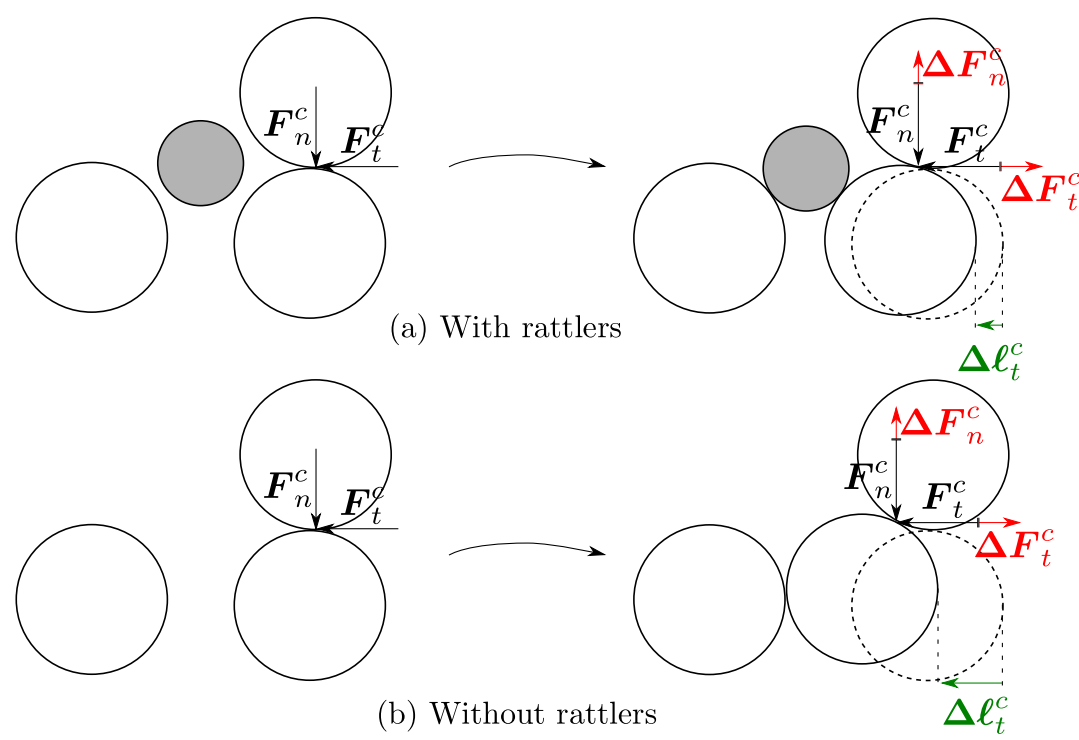

Figure 12: Schematic diagram of the particular role played by rattlers with respect to the size of the tangential increment $\left\|\Delta \ell_{t}^{c}\right\|$ for the incremental loading of Figure 11 (b).

thus a necessary condition to observe the vanishing of the local second-order work $w_{2}^{c}$. Then the vanishing of the second-order work $W_{2}$ at the scale of a representative elementary volume requires the following two conditions:

- A sufficient number of contacts have to slide through normal unloading;

- A large proportion of these contacts has to exhibit a sufficiently large tangential displacement $\Delta \ell_{t}^{c}$ such that the local second-order work $w_{2}^{c}$ vanishes.

By imposing internal geometrical constraints on a granular material, rattlers may contribute to limiting the size of the tangential increment $\left\|\Delta \ell_{t}^{c}\right\|$ when contact sliding occurs, as illustrated in Figure 12. Consequently, the presence of rattlers will result in a decrease in the number of contacts with $w_{2}^{c}<0$ and an increase in $W_{2}$, which imposes mechanical stability at the macroscopic scale. This constitutes a micromechanical proof of the conjecture formulated in Section 2.5. 


\section{A numerical inspection of the stabilizing role played by rattlers}

During the triaxial loading shown in Figure 2, two samples are saved for $\eta=0.35$ and $\eta=0.45$ (these states are marked with diamonds in Figure 2). As in Wautier et al. (2018) the mechanical stability of these two samples can be assessed with a stress-controlled directional analysis with stress probes describing a circle in the Rendulic plane $\left(\sqrt{2} \mathrm{~d} \sigma_{x x}, \mathrm{~d} \sigma_{z z}\right)$ as follows (see also Equation (3)):

$$
\left\{\begin{array}{cl}
\sqrt{2} \mathrm{~d} \sigma_{x x}=\sqrt{2} \mathrm{~d} \sigma_{y y} & =\|\mathrm{d} \boldsymbol{\sigma}\| \cos \theta \\
\mathrm{d} \sigma_{z z} & =\|\mathrm{d} \boldsymbol{\sigma}\| \sin \theta \\
\|\mathrm{d} \boldsymbol{\sigma}\| & =\sqrt{\mathrm{d} \sigma_{z z}^{2}+2 \mathrm{~d} \sigma_{x x}^{2}}
\end{array},\right.
$$

where $\|\mathrm{d} \boldsymbol{\sigma}\|=5 \mathrm{kPa}$ and $\theta \in[0,2 \pi]$.

To each stress probe $\mathrm{d} \boldsymbol{\sigma}$ corresponds an incremental strain response $\mathrm{d} \boldsymbol{\varepsilon}$. As

a result, the normalized second-order work $\bar{W}_{2}=\frac{\mathrm{d} \varepsilon: \mathrm{d} \boldsymbol{\sigma}}{\|\mathrm{d} \boldsymbol{\varepsilon}\|\|\mathrm{d} \boldsymbol{\sigma}\|}$ is a function of the loading direction $\theta$. In Figure 14, circular representations of $\bar{W}_{2}$ are shown for $\eta \in\{0.35,0.45\}$. As reviewed in the introduction, the vanishing of the second-order work is a signature of an underlying instability. As a result, the first state $\eta=0.35$ is identified as stable (at least in the Rendulic plane as $\left.\forall \theta \in[0,2 \pi], \bar{W}_{2}>0\right)$ whereas the second one $\eta=0.45$ corresponds to an unstable state with some incremental loading directions leading to the vanishing of the second-order work $\left(\forall \theta \in\left[205^{\circ}, 230^{\circ}\right], \bar{W}_{2}<0\right)$.

Based on these two samples, in this section we focus on the role played by the loose phase (i.e., the least stressed grains of the assembly) with respect to the mechanical stability of granular materials. In particular we focus on the role played by rattlers, i.e. particles with no contacts with their neighbors. Given that these particles do not carry any load, they can be easily removed from the samples saved, without decaying their bearing capacity. Similarly, rattlers can be artificially added to existing interstitial voids. These two artificial microstructure modifications will be considered in the following two subsections and may be representative of massive erosion or clogging induced by an internal flow.

\subsection{Mechanical stability assessment with no rattlers}

In Figure 13 (a) the sample saved in the mechanical state $\eta=0.45$ is shown with the free particles highlighted in white. In this case, artificial removal of 


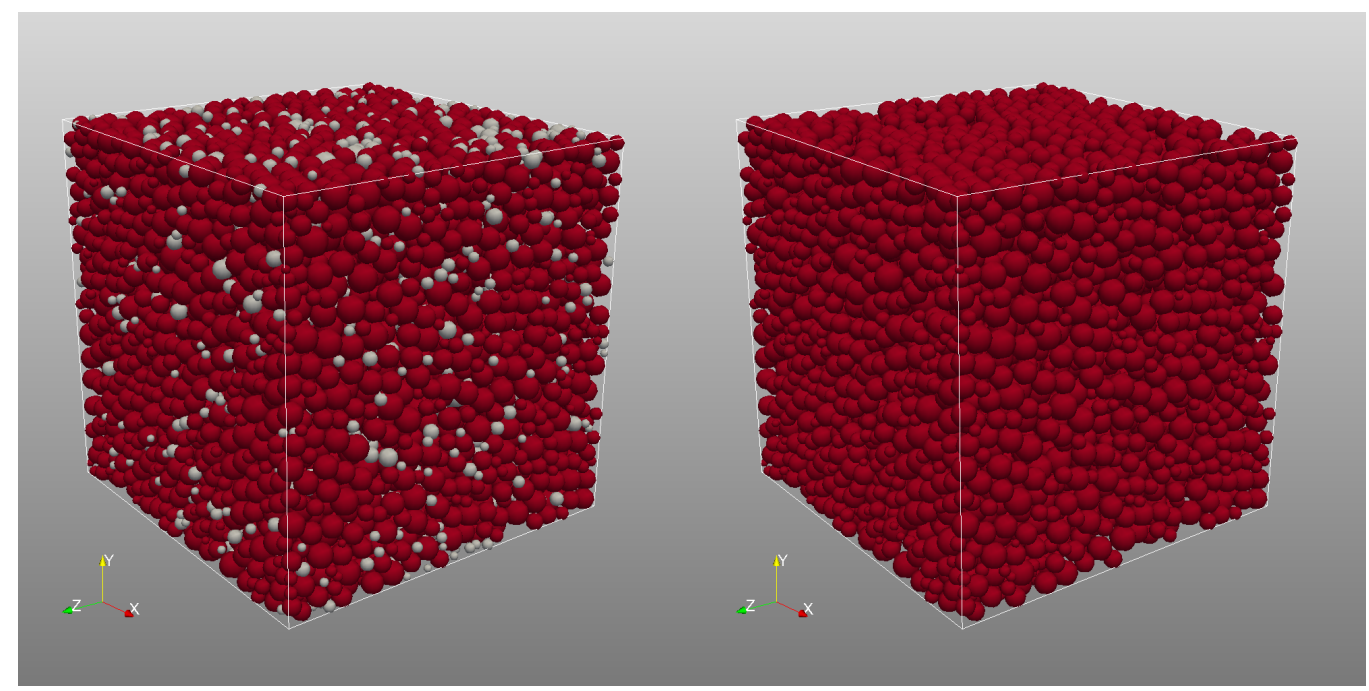

(a) with rattlers

(b) no rattlers

Figure 13: Microstructure evolution induced by removing free particles for the sample saved in the mechanical state $\eta=0.45$. Free particles are shown in the initial sample (a) in grey. After these 3480 particles are removed, the resulting microstructure is shown in (b) without rattlers.

rattlers concerns 3,480 particles out of 10,000 and the resulting microstructure is shown in Figure 13 (b). A similar extraction procedure was performed on the sample saved in the mechanical state $\eta=0.35$ in which 3,458 particles are found to be rattlers.

As for the virgin microstructure, a stress-controlled directional analysis (see Equation (3)) with 5 - $\mathrm{kPa}$ stress probes is performed with artificially eroded microstructures. In Figure 14, circular representations of the resulting normalized second-order work are shown for the two samples saved and compared with those obtained before any particle are removed.

In the case corresponding to $\eta=0.35$, the sample initially identified as stable with respect to the second-order work criterion $\left(\forall \theta, W_{2}>0\right)$ becomes unstable when rattlers are removed $\left(\exists \theta\right.$, such that $\left.W_{2}<0\right)$. In the case corresponding to $\eta=0.45$, both samples are in the bifurcation domain. Removing rattlers results in a wider cone of instability (the set of loading directions leading to $W_{2}<0$ ). These observations show that rattlers play an important role with respect to mechanical stability even though they did not initially contribute to supporting the mechanical load. Indeed, if the ex- 


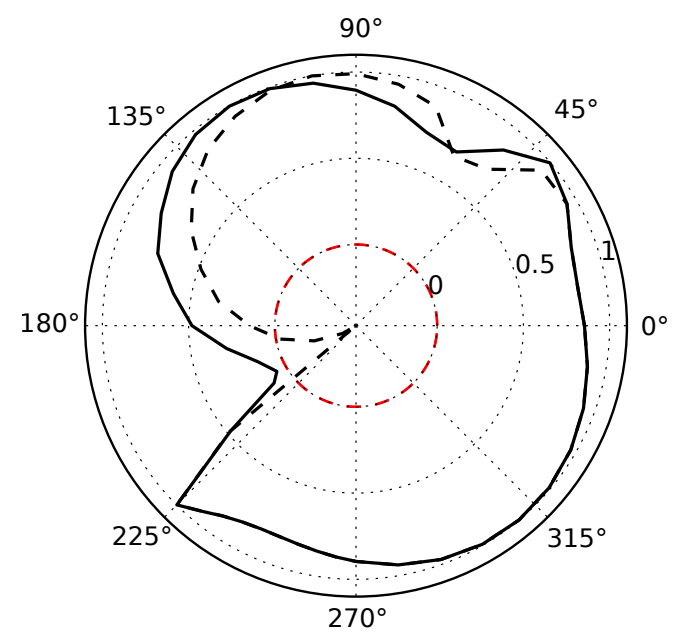

(a) $\eta=0.35$

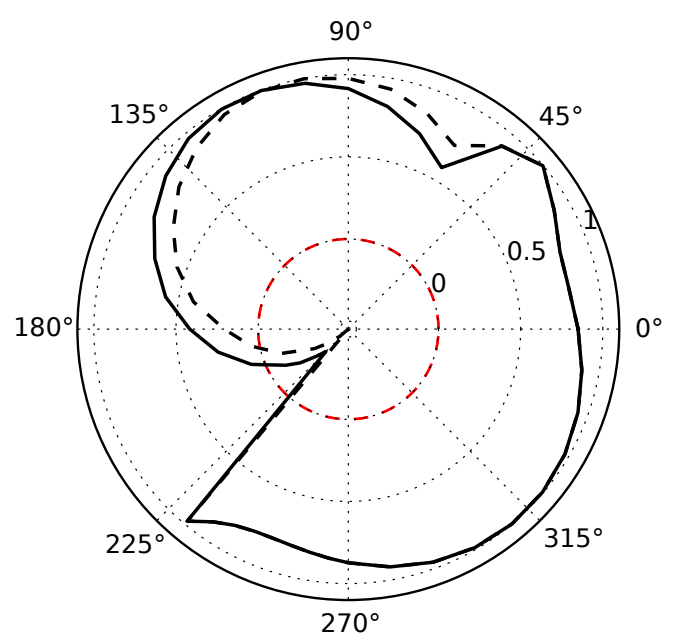

(b) $\eta=0.45$

Figure 14: Circular envelopes of the normalized second-order work for $\eta=0.35$ (a) and $\eta=0.45$ (b). The initial microstructure containing rattlers corresponds to the solid lines while the microstructure without rattlers corresponds to the dashed lines.

isting contact network fails to transmit the imposed incremental stress, the microstructure is forced to rearrange until a more robust contact network is built, possibly by mobilizing free particles. As a result, free particle removal reduces the rearrangement possibilities, which is consistent with an increase in the number of unstable loading directions. At the macroscale, this results in larger plastic increments. For instance, in the case of $\eta=0.45$, the maximum plastic intensity $\varepsilon_{p}$ introduced in section 2.3 rises from $4 \%$ to $21 \%$ when rattlers are removed. The corresponding incremental strain envelopes observed during the directional analysis performed are shown in Figure 17. These observations are consistent with previous studies in which particle removal is considered (including particles involved in stress transmission). For instance, Scholtès et al. (2010) showed that granular samples loaded in a drained triaxial state with a sufficiently high $\eta$ value cannot adapt to substantial particle removal. In some cases, removing some stressed particles is sufficient to induce the collapse. Indeed, destabilized contact networks cannot find any new stable configurations by incorporating new particles into force chains. Even if particle removal does not lead to instantaneous failure, Scholtès et al. (2010) and Hosn et al. (2018) observed considerable reduction of the peak stress in drained triaxial tests resulting from the decrease in the 
number of accessible microstructure configurations.

\subsection{Mechanical stability assessment with more rattlers}

Following the pore network analysis shown in Wautier et al. (2017), an artificial increase in the number of rattlers is achieved as follows:

- define a pore network using a regular Delaunay triangulation of the media (Chareyre et al., 2012);

- for every pore defined as a tetrahedron linking the center of four particles, compute the radius of the inscribed sphere together with the position of its center;

- check whether such a sphere intersects any other existing particles not belonging to the tetrahedron considered. If yes, decrease the computed inscribed sphere radius until no intersection exists;

- include a particle inside every pore where the inscribed radius is larger than a threshold value $r_{\text {th }}$.

The result of the above procedure with $r_{\text {th }}=\frac{r_{\min }}{2}$ is shown for the sample corresponding to $\eta=0.45$ in Figure 15 (b). A total of 12,805 particles have been added to existing voids (in addition to pre-existing rattlers).

As in the previous subsection, a stress-controlled directional analysis was performed with this artificially enriched microstructure. In Figure 16, circular representations of the normalized second-order work are shown for (i) the initial sample with $\eta=0.45$, (ii) this sample deprived from rattlers and (iii) this same sample in which additional rattlers are incorporated.

In Figure 16, the role played by rattlers with respect to mechanical stability is clearly shown and confirms the conjecture proposed in Section 2.5. Indeed, adding new rattlers offers new possibilities in rebuilding contact networks. In the case of $\eta=0.45$, the maximum plastic intensity $\varepsilon_{p}$ is reduced from $4 \%$ to $0.8 \%$ when additional rattlers are considered, as shown in Figure 17. As a result, fewer incremental loading directions are associated with a negative value of the second-order work as observed in Figure 16. However, despite the large number of newly incorporated rattlers, a cone of instability persists for the sample considered. These additional rattlers do not get jammed into a new contact network quickly enough to prevent the existence of macroscopic instability. 


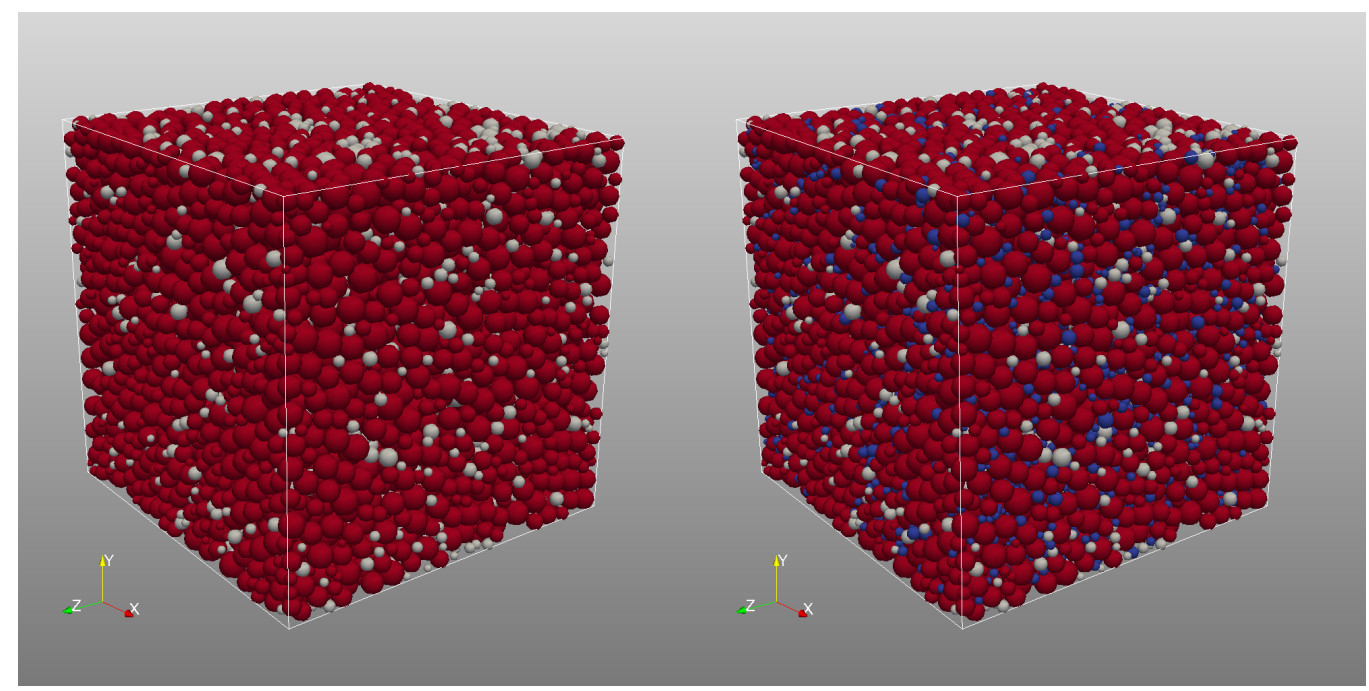

(a) with rattlers

(b) more rattlers

Figure 15: Microstructure evolution induced by inclusion of free particles for the sample saved in the mechanical state $\eta=0.45$. Free particles are shown in the initial sample (a) in white. The 12,805 particles added are shown in blue in the enriched sample (b).

\subsection{Impact of rattlers on the non-associated character of the flow rule}

As shown in the previous subsection, rattlers have a significant impact on the mechanical stability of the samples considered. For the sample corresponding to $\eta=0.45$ it is interesting to reconsider the model parameters of Table 2 while updating the plastic flow intensity $\varepsilon_{p}$ to account for the removal or addition of rattlers considered in this section $\left(\varepsilon_{p}=2110^{-2}\right.$ and $\varepsilon_{p}=$ $810^{-3}$ respectively). The comparison between the resulting envelopes and the numerical data is given in Figure 18.

As shown in Figure 18, the change in the plastic intensity explains most of the change in the $W_{2}$ circular envelope in the case with additional rattlers but does not account for the modification induced by the removal of rattlers. Indeed, the presence or absence of rattlers may have an other impact on the plastic behavior at the continuum scale through the plastic flow direction as illustrated in the schematic diagram of Figure 19. The macroscopic activation of the plastic behavior corresponds locally to substantial grain rearrangements following the collapse of pre-existing force chains. Initially these force chains are oriented in the principal stress direction along the $z$ axis. Once they fail, the sample contracts in this direction while slightly dilating in the 


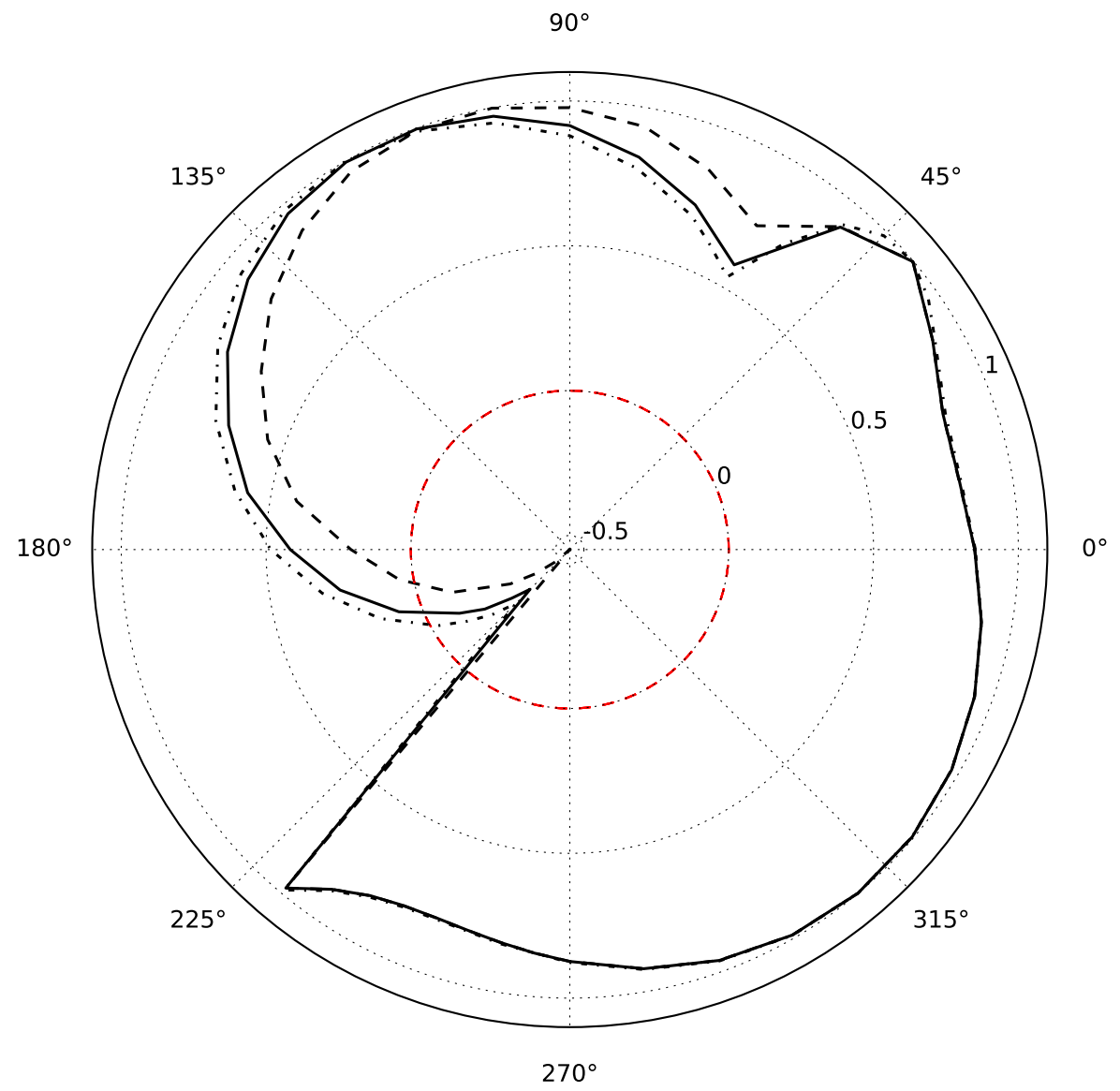

Figure 16: Circular envelopes of normalized second-order work for $\eta=0.45$. Three microstructures are considered: the initial microstructure (solid line), the initial microstructure without its rattlers (dashed line) and the initial microstructure enriched with more rattlers (dotted-dashed line). 


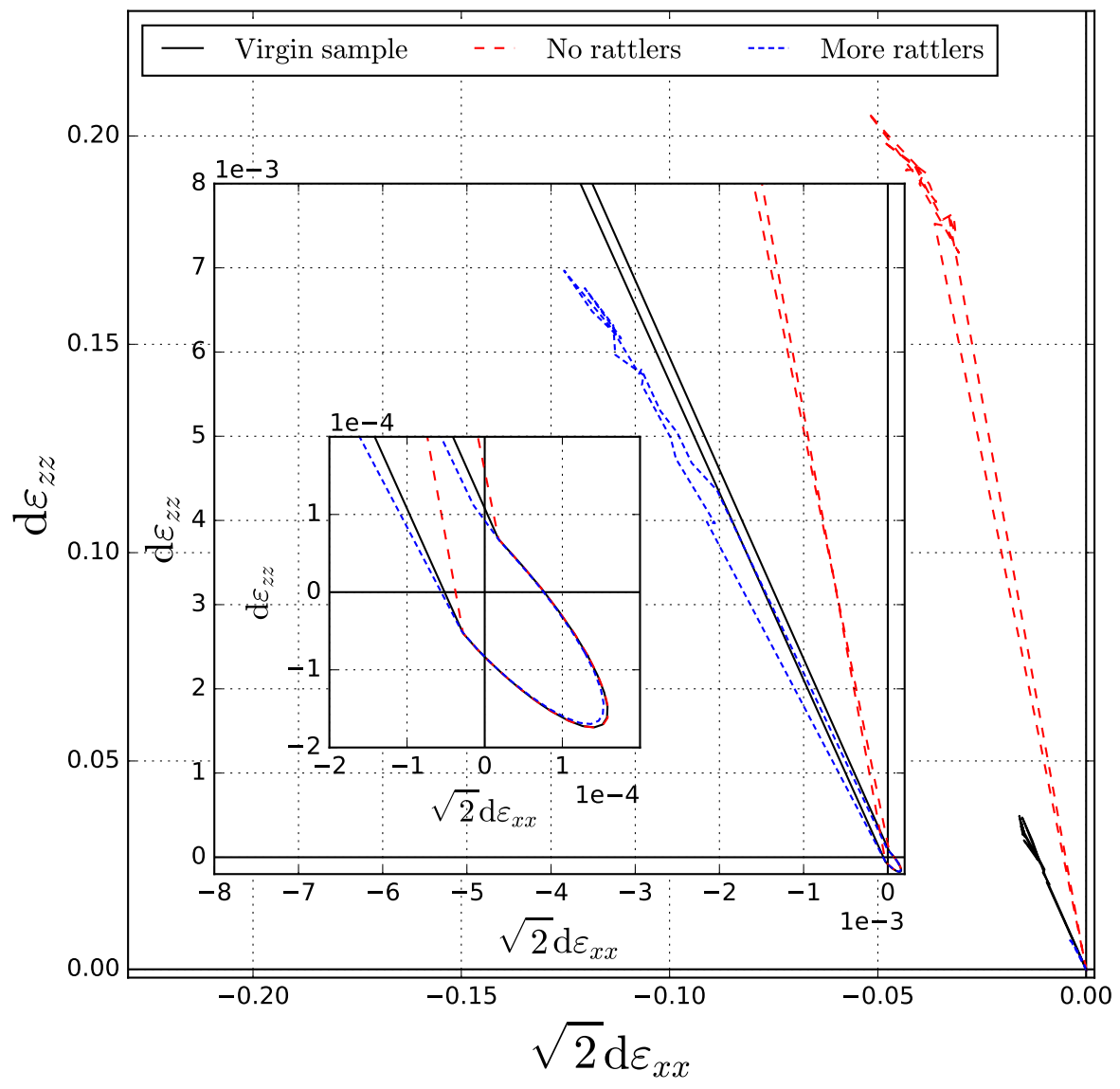

Figure 17: Incremental strain envelopes associated with directional analyses in the case of $\eta=0.45$. The three microstructures shown in Figures 13 and 15 are considered: virgin (solid line), with rattlers removed (large dashed line), with rattlers added (small dashed line). Two levels of zoom are provided in the inset plots. 


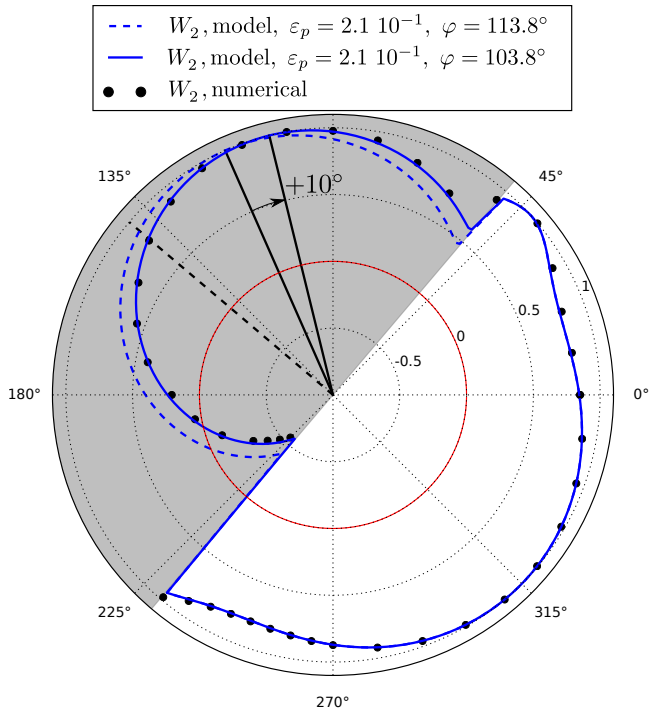

(a) No rattlers

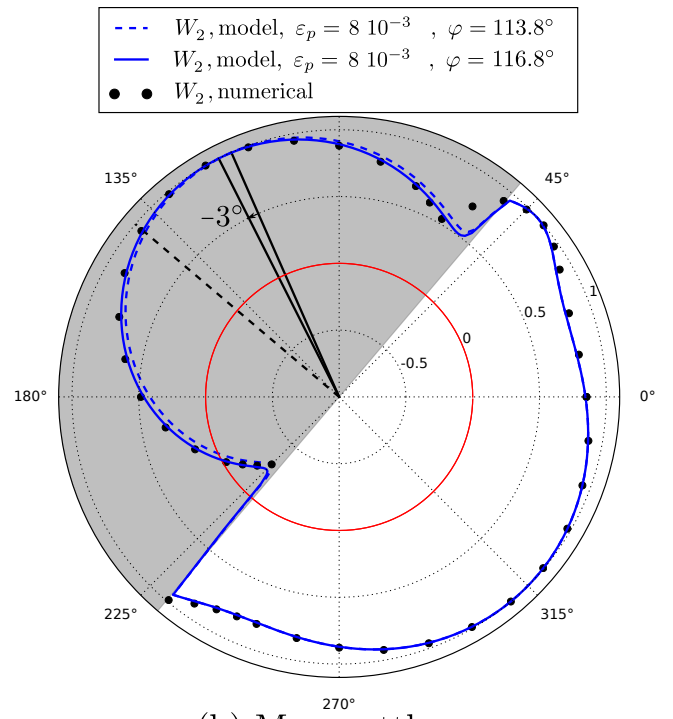

(b) More rattlers

Figure 18: Circular envelopes of normalized second-order work for $\eta=0.45$ corresponding to the sample deprived from rattlers (a) and in which additional rattlers are incorporated (b). The phenomenological fits coming from the elastoplastic model presented in Section 2.3 are shown with a dashed line with parameters from Table 2 and updated $\varepsilon_{p}$ values, and as a solid line for the updated plastic parameters from Table3. The maximum plastic intensity direction $\varphi+\Delta \varphi$ (the black dashed line segment) and the plastic flow direction $\varphi$ (the two black solid line segments) are also shown for both fits. 


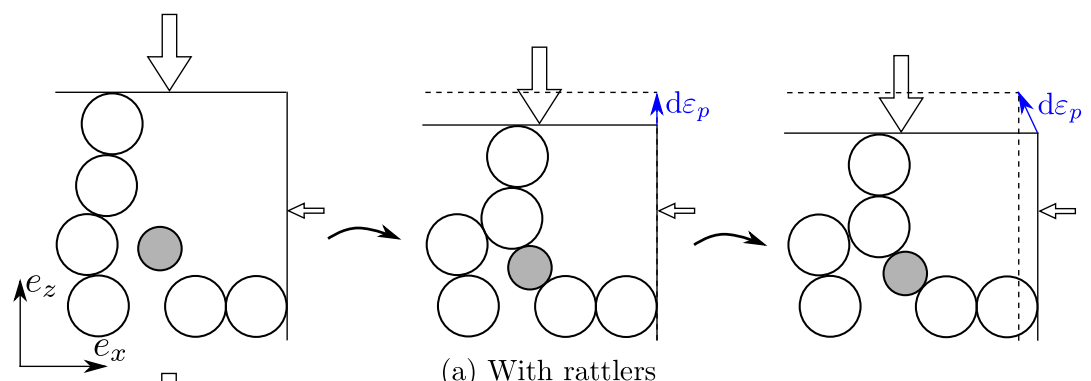

(a) With rattlers
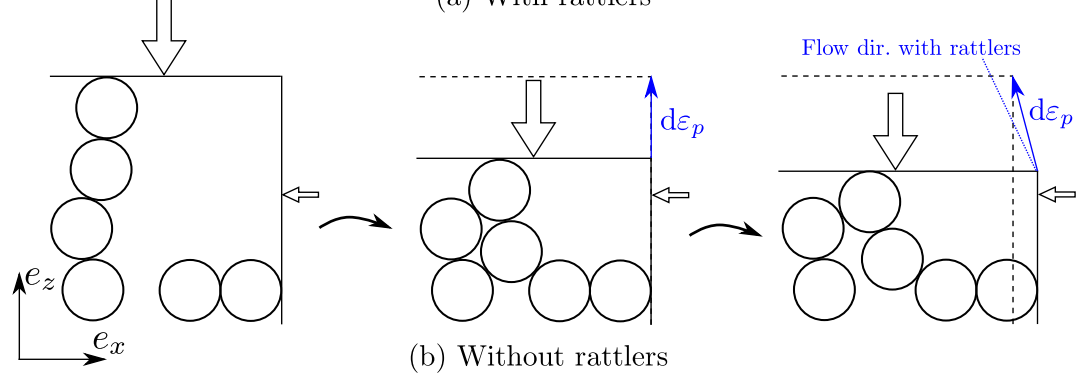

(b) Without rattlers

Figure 19: Illustration of the plastic flow rotation induced by rattler removal with anisotropically oriented force chains in the $z$ direction.

horizontal directions $x$ and $y$ as a consequence of vertical to lateral force transmission. When rattlers are removed, the vertical contraction is stopped later while the horizontal dilation is less influenced. Since incremental plastic strains result from the collapse of mesostructures mostly oriented along the $z$ axis, the impact of rattlers is expected to be greater along $\mathrm{d} \varepsilon_{z z}$ than along $\mathrm{d} \varepsilon_{x x}$ and $\mathrm{d} \varepsilon_{y y}$. This asymmetric influence results in a clockwise rotation of the plastic flow in the axisymmetric plane $\left(\sqrt{2} \mathrm{~d} \varepsilon_{x x}, \mathrm{~d} \varepsilon_{z z}\right)$ as observed numerically in Figure 17. However, since the onset of the plastic behavior is governed by the primary fabric, the plastic half-plane is not affected by the modification of the population of rattlers.

As shown in Figure 18, a decrease in the plastic flow direction $\varphi$ while keeping $\varphi+\Delta \varphi$ constant precisely accounts for the new circular envelopes when rattlers are removed from the sample. On the contrary, when adding rattlers, a small increase in the plastic flow direction $\varphi$ provides a better fit of the numerical data. The modified plastic parameters are given in Table 3 
Table 3: Updated plastic parameters for the incremental non-associated elastoplastic behavior for the mechanical state $\eta=0.45$ when rattlers are removed or added.

\begin{tabular}{l|l} 
No rattlers & More rattlers \\
\hline$\varphi=113.8^{\circ}-10^{\circ}$ & $\varphi=113.8^{\circ}+3^{\circ}$ \\
$\Delta \varphi=25.9^{\circ}+10^{\circ}$ & $\Delta \varphi=25.9^{\circ}-3^{\circ}$ \\
$\varepsilon_{p}=2110^{-2}$ & $\varepsilon_{p}=810^{-3}$
\end{tabular}

\section{Conclusion and outlook}

The combination of phenomenological modeling at the continuum scale, microto macro-analytical relations and discrete element simulations was used in this paper to elucidate the micro-mechanisms responsible for the macroscopic concepts of plasticity and material instability. In this respect, particular attention was paid to provide physical interpretations of the phenomenological parameters used in non-associated elasto-plastic constitutive modeling. In particular, the role played by free particles in granular materials has been investigated in detail.

A necessary condition for the occurrence of material instability within granular materials (detected by the second-order work criterion) lies in the development of large plastic strains. Provided that the current contact network fails to withstand an incremental load, the mechanical stability of a granular material at the macroscale is closely related to the ability of free particles to get jammed into new force chains. Indeed, removing free particles results in an increase in incremental plastic strain. When the loading history induces microstructure anisotropy, local microstructure rearrangements occur in a privileged direction, which macroscopically affects the direction of the flow rule. As a consequence of these two microstructure effects, removing rattlers leads to an increase in the number of loading directions associated with a vanishing second-order work. On the other hand, adding free particles inside the pores of an unstable granular assembly is shown to have a stabilizing effect.

The understanding of such micromechanisms is of paramount importance while considering the development of micromechanical models (Chang and Hicher, 2005; Zhu et al., 2006; La Ragione et al., 2008; Zhu et al., 2010; Nicot and Darve, 2011; Xiong et al., 2017). In these "bottom-up" models, and contrary to macroscopic phenomenological models, no assumptions are made a priori on the form of the overall constitutive behavior, which will sim- 
ply result from the micromechanisms captured in a simplified manner at the micro or mesoscale. As a result, micromechanical models in which mesostructures are rich enough to build anisotropic microstructures will easily capture the anisotropic elastic behavior visible at the representative elementary volume in this paper. If these mesostructures can account for the collapse and rebuilding of force chains incorporating free particles (the phase exchange between strong and weak contact networks), the resulting models will certainly be able to account for plastic strain intensity and capture the resulting mechanical instability. In addition, if these mesostructures can evolve under external couplings (fluid/grain couplings, chemical deposition or dissolution, etc.), the micromechanical models considered will be able to account for the induced evolutions in terms of the mechanical stability of a great diversity of processes at stake inside granular materials.

In this respect, the results of this paper have a particular significance related to internal erosion processes, and in particular the selective erosion of the fine particles of a soil also known as suffusion. If candidate particles to erosion are too large to be transported through the pore space, they remain trapped inside the soil and ensure mechanical stability. In contrast, if the soil loses rattlers, mechanical instability is more likely to appear. The development of enriched versions of micromechanical models such as the one proposed by Nicot and Darve (2011) and recently extended in 3D by Xiong et al. (2017) could therefore lead to advanced constitutive modeling of internal processes in soils for engineering applications in which detecting underlying mechanical instability is of paramount importance. To pave the way for developing such models, a closer perspective lies in the numerical modeling of fluid/grain interactions at the microscale to provide a more precise picture of flow-induced microstructure modifications. 


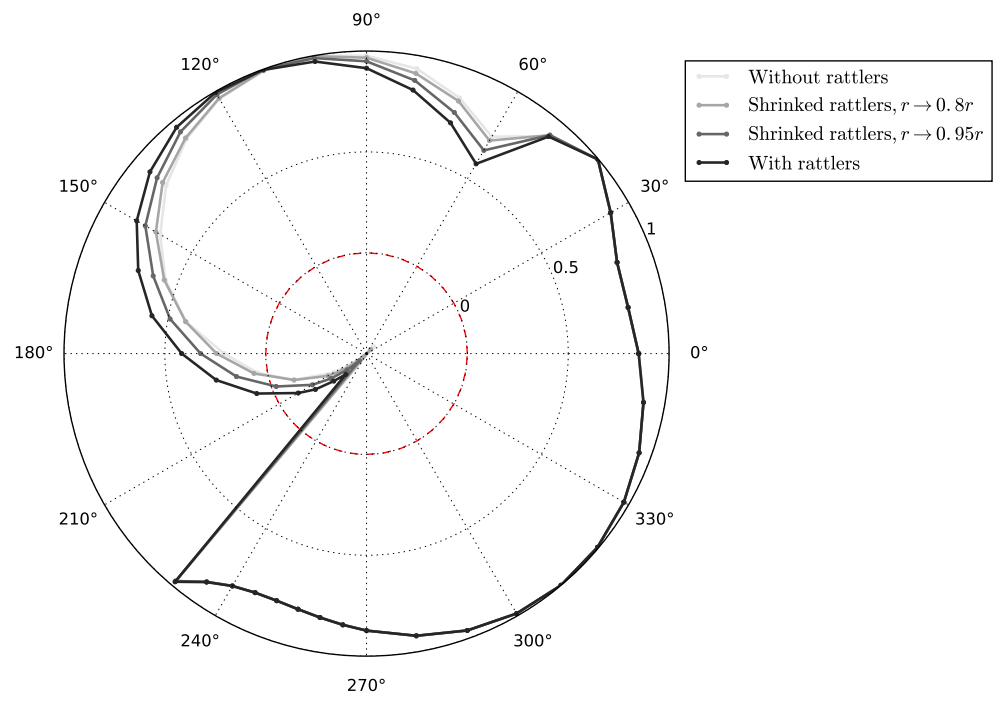

Figure A.20: Second-order work circular envelopes for different size reduction of rattlers. The used sample corresponds to the stress state $\eta=0.45$. Four size reduction factors are considered $(1,0.95,0.8$ and 0$)$.

\section{Appendix A. DEM inspection of contact scale influence of rattlers}

The objective of Section 3 was to exhibit the contact scale mechanisms responsible for material instability in order to prove the conjecture formulated in Section 2.5. In this section, complementary DEM simulations are considered to back the qualitative results of Section 3 with some quantitative analyses. In these DEM simulations, the sample corresponding to the stress state $\eta=0.45$ is considered, and rattlers are not removed but shrunk by a factor of 0.95 or 0.8 . The circular envelopes corresponding to these new microstructures are shown in Figure A.20 together with those of Figure 14 (b).

In Figure A.20, the size influence of rattlers is visible as the instability cone progressively widens when rattlers are shrunk which is consistent with the schematic diagrams of Figures 10, 12 and 19.

If grain rotations are neglected for the sake of simplicity, the incremental relative displacements between grains can be recovered by comparing the branch vectors in the final (after the application of an incremental loading) and initial configurations for every couple of particles initially in contact. By taking the initial branch vector as reference, the final branch vector can be decomposed into a normal displacement $\Delta \boldsymbol{u}_{n}$ and a tangential displacement 
$\Delta \boldsymbol{u}_{t}$. For each vector $\Delta \boldsymbol{u}_{n}$, a scalar quantity $\Delta u_{n}$ is defined with $\Delta u_{n}<$ 0 corresponding to a decrease in the normal inter-penetration and $\Delta u_{n}>$ 0 corresponding to an increase in the normal inter-penetration. In Figure A.21, the observed incremental displacements between all particles initially in contact are shown for the sample corresponding to the stress state $\eta=0.45$ when rattlers are not shrunk, when rattlers are shrunk by a factor 0.95 and when rattlers are shrunk by a factor 0.8 .

In Figure A.21, a lot of relative displacements correspond to normal unloading $\left(\Delta u_{n}<0\right)$ which is the contact scale necessary condition recalled in Section 3 to observe the macroscopic vanishing of the second-order work. While rattlers are shrunk, $\left\|\Delta \boldsymbol{u}_{t}\right\|$ tends to increases. This tendency is confirmed in Figure A.22 in which the tangential displacements of contacts undergoing normal unloading in the reference case (for which rattlers are not shrunk) are compared before and after rattlers are shrunk by $5 \%$. Results are normalized by the mean particle radius $r_{\text {mean }}$ corresponding to the reference sample in which particles are not shrunk.

In Figure A.22, the mechanism detailed in Figure 12 is observed in DEM simulations. The majority of contacts subjected to normal unloading when rattlers are not shrunk continue to experience normal unloading when rattlers are shrunk by $5 \%$. In addition, for these contacts, the tangential displacements increase as rattlers are shrunk. These quantitative results are in agreement with the analytical findings of Section 3. 

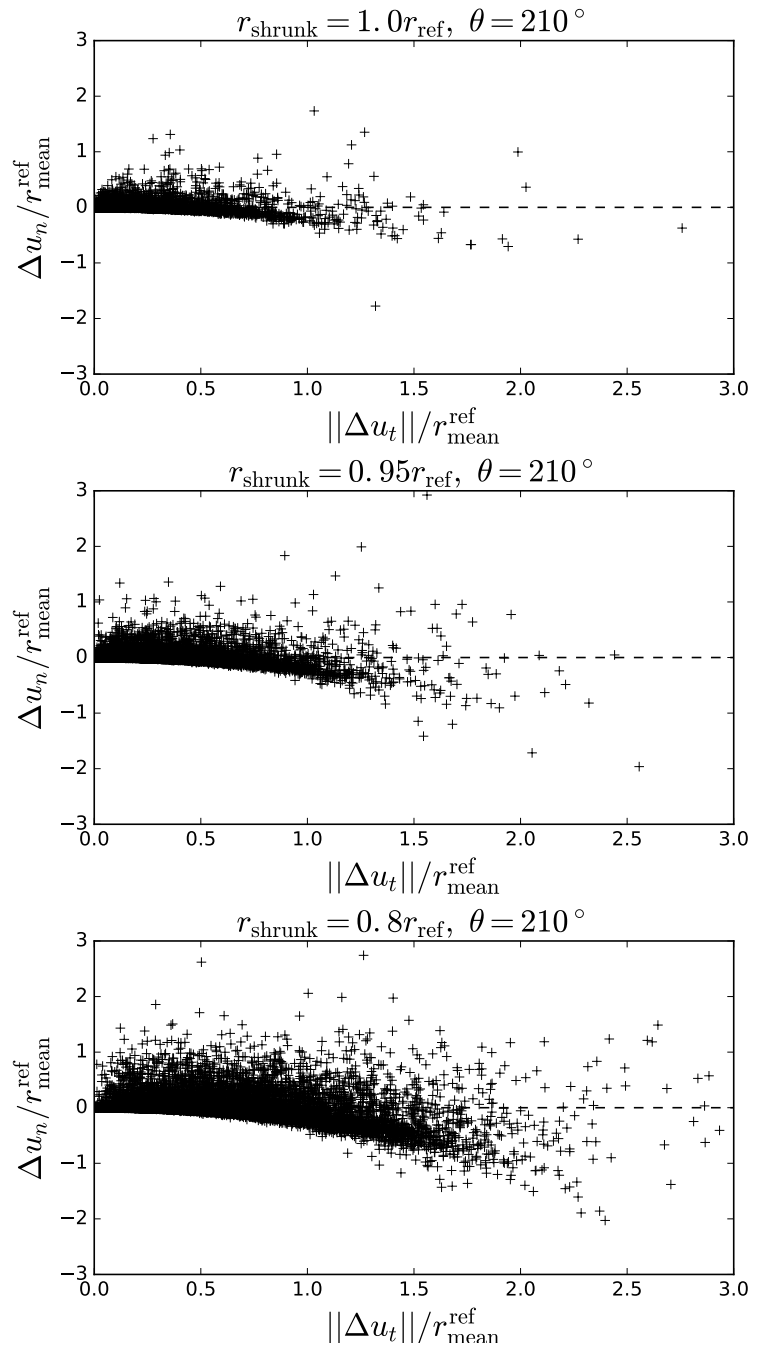

Figure A.21: Incremental displacements $\Delta u_{n}$ and $\left\|\Delta \boldsymbol{u}_{t}\right\|$ between all particles initially in contact for the sample corresponding to the stress state $\eta=0.45$ subjected to an incremental stress load leading to the vanishing of the second-order work criterion (see Figure A.20). Three size reduction factors are considered (1,0.95 and 0.8). Results are normalized by the mean particle radius $r_{\text {mean }}$ corresponding to the reference sample in which particles are not shrunk. 


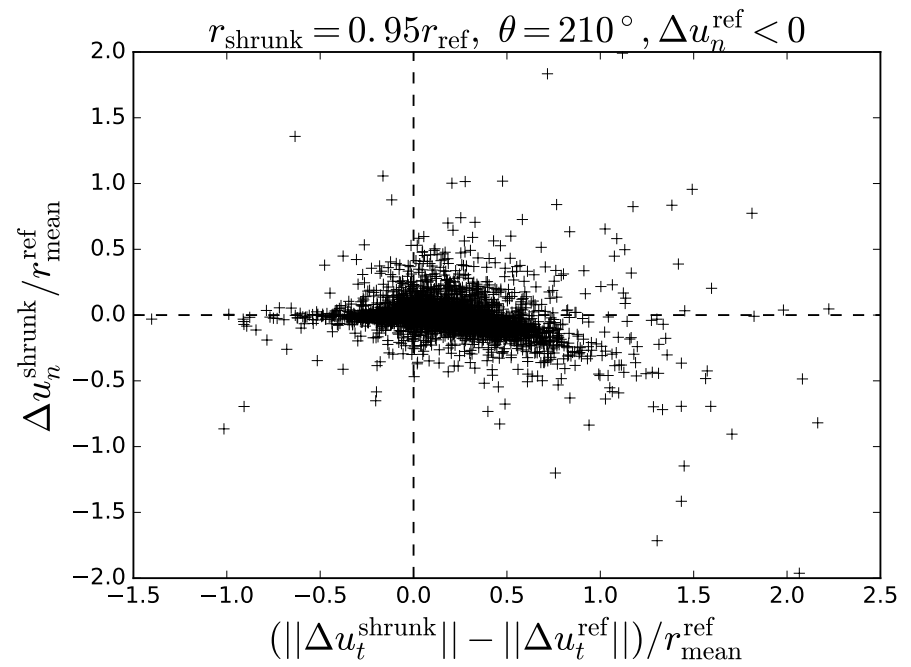

Figure A.22: Incremental displacements $\Delta u_{n}^{\text {shrunk }}$ and $\left\|\Delta \boldsymbol{u}_{t}^{\text {shrunk }}\right\|-\left\|\Delta \boldsymbol{u}_{t}^{\text {ref }}\right\|$ between all particles initially in contact for the sample corresponding to the stress state $\eta=0.45$ subjected to an incremental stress load leading to the vanishing of the second-order work criterion (see Figure A.20). Three size reduction factors are considered (1, 0.95 and 0.8$)$. Results are normalized by the mean particle radius $r_{\text {mean }}$ corresponding to the reference sample in which particles are not shrunk. 


\section{References}

Bardet, J.-P., 1994. Numerical simulations of the incremental responses of idealized granular materials. International Journal of Plasticity 10 (8), 879908.

Bigoni, D., Hueckel, T., 1991. Uniqueness and localization - i. associative and non-associative elastoplasticity. International Journal of Solids and structures 28 (2), 197-213.

Calvetti, F., 2003. A numerical investigation of the incremental behavior of granular soils. Revista Italiana di Geotechnica 37, 5-23.

Challamel, N., Nicot, F., Lerbet, J., Darve, F., 2010. Stability of nonconservative elastic structures under additional kinematics constraints. Engineering Structures 32 (10), 3086-3092.

Chang, C. S., Hicher, P.-Y., 2005. An elasto-plastic model for granular materials with microstructural consideration. International journal of solids and structures 42 (14), 4258-4277.

Chareyre, B., Cortis, A., Catalano, E., Barthélemy, E., 2012. Pore-scale modeling of viscous flow and induced forces in dense sphere packings. Transport in porous media $94(2), 595-615$.

Cundall, P. A., Strack, O. D., 1979. A discrete numerical model for granular assemblies. Geotechnique 29 (1), 47-65.

Daouadji, A., Darve, F., Al Gali, H., Hicher, P., Laouafa, F., Lignon, S., Nicot, F., Nova, R., Pinheiro, M., Prunier, F., et al., 2011. Diffuse failure in geomaterials: experiments, theory and modelling. International Journal for Numerical and Analytical Methods in Geomechanics 35 (16), 17311773 .

Darve, F., Laouafa, F., 2000. Instabilities in granular materials and application to landslides. Mechanics of Cohesive-frictional Materials 5 (8), 627652 .

Dean, E., 2005. Patterns, fabric, anisotropy, and soil elasto-plasticity. International journal of plasticity 21 (3), 513-571. 
Drucker, D. C., Prager, W., 1952. Soil mechanics and plastic analysis or limit design. Quarterly of applied mathematics 10 (2), 157-165.

Griffiths, D., Willson, S., 1986. An explicit form of the plastic matrix for a mohr-coulomb material. International Journal for Numerical Methods in Biomedical Engineering 2 (5), 523-529.

Gudehus, G., 1979. A comparison of some constitutive laws for soils under radially symmetric loading and unloading. Canadian Geotechnical Journal $20,502-516$.

Hadda, N., Nicot, F., Bourrier, F., Sibille, L., Radjai, F., Darve, F., 2013. Micromechanical analysis of second order work in granular media. Granular matter 15 (2), 221-235.

Hill, R., 1958. A general theory of uniqueness and stability in elastic-plastic solids. Journal of the Mechanics and Physics of Solids 6 (3), 236-249.

Hosn, R. A., Sibille, L., Benahmed, N., Chareyre, B., 2018. A discrete numerical model involving partial fluid-solid coupling to describe suffusion effects in soils. Computers and Geotechnics 95, 30-39.

Kishino, Y., 2003. On the incremental nonlinearity observed in a numerical model for granular media. Italian Geotechnical Journal 3, 3-12.

La Ragione, L., Prantil, V. C., Sharma, I., 2008. A simplified model for inelastic behavior of an idealized granular material. International Journal of Plasticity 24 (1), 168-189.

Lai, Y., Jin, L., Chang, X., 2009. Yield criterion and elasto-plastic damage constitutive model for frozen sandy soil. International Journal of Plasticity 25 (6), 1177-1205.

Li, M., Richmond, O., 1997. Intrinsic instability and nonuniformity of plastic deformation. International journal of plasticity 13 (8-9), 765-784.

Li, Y., Karr, D. G., 2009. Prediction of ductile fracture in tension by bifurcation, localization, and imperfection analyses. International Journal of Plasticity 25 (6), 1128-1153.

Liu, C., Nagel, S. R., Schecter, D., Coppersmith, S., Majumdar, S., et al., 1995. Force fluctuations in bead packs. Science 269 (5223), 513. 
Nicot, F., Darve, F., 2006a. Micro-mechanical investigation of material instability in granular assemblies. International Journal of Solids and Structures 43 (11), 3569-3595.

Nicot, F., Darve, F., 2006b. On the elastic and plastic strain decomposition in granular materials. Granular Matter 8 (3-4), 221-237.

Nicot, F., Darve, F., 2007a. Basic features of plastic strains: from micromechanics to incrementally nonlinear models. International Journal of Plasticity 23 (9), 1555-1588.

Nicot, F., Darve, F., 2007b. Micro-mechanical bases of some salient constitutive features of granular materials. International Journal of Solids and Structures 44 (22), 7420-7443.

Nicot, F., Darve, F., 2011. The h-microdirectional model: accounting for a mesoscopic scale. Mechanics of Materials 43 (12), 918-929.

Nicot, F., Hadda, N., Darve, F., 2013. Second-order work analysis for granular materials using a multiscale approach. International Journal for $\mathrm{Nu}$ merical and Analytical Methods in Geomechanics 37 (17), 2987-3007.

Nicot, F., Lerbet, J., Darve, F., 2017. Second-order work criterion: from material point to boundary value problems. Acta Mechanica, 1-16.

Nicot, F., Sibille, L., Darve, F., 2009. Bifurcation in granular materials: An attempt for a unified framework. International Journal of Solids and Structures 46 (22), 3938-3947.

Nicot, F., Sibille, L., Darve, F., 2012. Failure in rate-independent granular materials as a bifurcation toward a dynamic regime. International Journal of Plasticity 29, 136-154.

Nicot, F., Sibille, L., Donze, F., Darve, F., 2007. From microscopic to macroscopic second-order work in granular assemblies. Mechanics of materials 39 (7), 664-684.

Nicot, F., Veylon, G., Huaxiang, Z., Lerbet, J., Darve, F., 2016. Mesoscopic scale instability in particulate materials. Journal of Engineering Mechanics 142 (8), 04016047. 
Peters, J. F., Muthuswamy, M., Wibowo, J., Tordesillas, A., 2005. Characterization of force chains in granular material. Physical review E 72 (4), 041307.

Radjai, F., Wolf, D. E., Jean, M., Moreau, J.-J., 1998. Bimodal character of stress transmission in granular packings. Physical review letters 80 (1), 61.

Sandler, I. S., Rubin, D., 1987. The consequences of non-associated plasticity in dynamic problems. Constitutive Laws for Engineering Materials, 345352.

Scholtès, L., Hicher, P.-Y., Sibille, L., 2010. Multiscale approaches to describe mechanical responses induced by particle removal in granular materials. Comptes Rendus Mécanique 338 (10), 627-638.

Sibille, L., Nicot, F., Donzé, F.-V., Darve, F., 2009. Analysis of failure occurrence from direct simulations. European Journal of Environmental and Civil Engineering 13 (2), 187-201.

Stoughton, T. B., Yoon, J. W., 2008. On the existence of indeterminate solutions to the equations of motion under non-associated flow. International Journal of Plasticity 24 (4), 583-613.

Tashman, L., Masad, E., Little, D., Zbib, H., 2005. A microstructure-based viscoplastic model for asphalt concrete. International Journal of Plasticity 21 (9), 1659-1685.

Tordesillas, A., Walker, D. M., Lin, Q., 2010. Force cycles and force chains. Physical Review E 81 (1), 011302.

Šmilauer et al., V., 2015. Yade Documentation 2nd ed. The Yade Project, http://yade-dem.org/doc/.

Wan, R., Nicot, F., Darve, F., 2016. Failure in Geomaterials, a contemporary treatise. ISTE/Elsevier.

Wan, R., Pinheiro, M., Daouadji, A., Jrad, M., Darve, F., 2013. Diffuse instabilities with transition to localization in loose granular materials. International Journal for Numerical and Analytical Methods in Geomechanics 37 (10), 1292-1311. 
Wautier, A., Bonelli, S., Nicot, F., 2017. Scale separation between grain detachment and grain transport in granular media subjected to an internal flow. Granular Matter 19 (2), 22.

Wautier, A., Bonelli, S., Nicot, F., 2018. Micro-inertia origin of instabilities in granular materials. International Journal for Numerical and Analytical Methods in Geomechanics 42 (9), 1037-1056.

Xiong, H., Nicot, F., Yin, Z., 2017. A three-dimensional micromechanically based model. International Journal for Numerical and Analytical Methods in Geomechanics 41 (17), 1669-1686.

Yang, Y., Yu, H., Muraleetharan, K., 2005. Solution existence conditions for elastoplastic constitutive models of granular materials. International journal of plasticity 21 (12), 2406-2425.

Yuanming, L., Yugui, Y., Xiaoxiao, C., Shuangyang, L., 2010. Strength criterion and elastoplastic constitutive model of frozen silt in generalized plastic mechanics. International Journal of Plasticity 26 (10), 1461-1484.

Zhang, L., Nguyen, N. G. H., Lambert, S., Nicot, F., Prunier, F., DjeranMaigre, I., 2016a. The role of force chains in granular materials: from statics to dynamics. European Journal of Environmental and Civil Engineering, 1-22.

Zhang, X., Zhang, X., Shang, F., Li, Q., 2016b. Second-order work and strain burst in single-crystalline micropillar plasticity. International Journal of Plasticity 77, 192-213.

Zhu, H., Mehrabadi, M. M., Massoudi, M., 2006. Three-dimensional constitutive relations for granular materials based on the dilatant double shearing mechanism and the concept of fabric. International journal of plasticity $22(5), 826-857$.

Zhu, H., Nguyen, H. N., Nicot, F., Darve, F., 2016a. On a common critical state in localized and diffuse failure modes. Journal of the Mechanics and Physics of Solids 95, 112-131.

Zhu, H., Nicot, F., Darve, F., 2016b. Meso-structure organization in twodimensional granular materials along biaxial loading path. International Journal of Solids and Structures 96, 25-37. 
Zhu, Q., Shao, J.-F., Mainguy, M., 2010. A micromechanics-based elastoplastic damage model for granular materials at low confining pressure. International Journal of Plasticity 26 (4), 586-602.

Zhu, Q., Zhao, L., Shao, J., 2016c. Analytical and numerical analysis of frictional damage in quasi brittle materials. Journal of the Mechanics and Physics of Solids 92, 137-163. 